\title{
Molecular Dynamic Simulations and Binding Free Energy Evaluations of Thiazolo-[2,3-b] Quinazolinone Derivatives With wtEGFR-TKD and "TMLR" Mutant EGFR-TKD
}

Showkat Mir ( $\square$ showkat@suniv.ac.in )

Sambalpur University

Binata Nayak

Sambalpur University

\section{Research Article}

Keywords: wtEGFR-TKD, EGFR-TKD mutant "TMLR", Thiazolo-[2,3-b] quinazolinone derivatives, Molecular Dynamic Simulations, MM-PBSA

Posted Date: November 23rd, 2021

DOI: https://doi.org/10.21203/rs.3.rs-1059565/v1

License: (c) (i) This work is licensed under a Creative Commons Attribution 4.0 International License. Read Full License 


\section{Abstract}

Cancer causes innumerable deaths every year globally. Breast cancer and non-small cell lung carcinoma (NSCLC) are the most prevalent worldwide. The epidermal growth factor receptor tyrosine kinases play a pivotal role in manifestations of cellular signals in carcinoma cells and thus are endorsed as therapeutic targets in cancer management. EGFR-TKD is a good option for the treatment of cancer, but the resistance shown by first-generation TKIs leads to hyperphosphorylation, overexpression, and mutations of EGFR-TKD. The new molecular scaffolds of thiazolo-[2,3-b] quinazolinones were evaluated against EGFR-TKD via molecular docking simulations and thereby linked with molecular dynamic simulations to identify the ligand stability with EGFR-TKD's. The binding energy of thiazolo-[2,3-b] quinazolinones is approximately similar to that of reference molecules found against both wild type wtEGFR-TKD and EGFR-TKD mutant "TMLR" (T790M/L858R) in this study. According to ADMET analysis, thiazolo$[2,3-b]$ quinazolinone derivatives (5ab, 5aq, and $5 \mathrm{bq}$ ) are safe. The stability was investigated at an atomistic level by molecular dynamic simulations, and the strength of the bindings was calculated by the molecular mechanics Poisson-Boltzmann surface areas continuum solvation MM-PBSA method. The RMSD, radius of gyration, and SASA trajectories were studied in detail. The $\Delta$ Gbind generated by heterocyclic 5aq thiazolo-[2,3-b] quinazolinone was found to be $-63.723 \pm 0.419 \mathrm{~kJ} / \mathrm{mol}$ against the EGFR-TKD mutant "TMLR" and it was $-51.551 \pm 0.409 \mathrm{~kJ} / \mathrm{mol}$ against wtEGFR-TKD. This study concludes that the top ranked complexes 5ab, 5aq, and 5bq with both wild and mutated types of EGFR-TKD corroborate that thiazolo-[2,3-b] quinazolinone derivatives, like the FDA-approved drug erlotinib, may be potent inhibitors of EGFR-TKD for patients with NSCLC.

\section{Introduction:}

Cancer is the second most deadly disease around the globe, and in the year 2019, 9.6 million deaths occurred due to cancer $^{1}$. The number of new cancer cases is expected to be $1,898,160$ with 608,570 deaths in the United States alone in $2021^{2}$ and propagates widely in underdeveloped nations. Cancer accounts for $70 \%$ of deaths, which is one in every six deaths ${ }^{3}$. Many transcriptional factors facilitate abnormal growth, recruit many signalling cascades together, and result in proliferation, angiogenesis, metastasis, and cell death ${ }^{4}$. The alteration of normal metabolism and functionality results in abnormal growth of cells ${ }^{5}$ and growth beyond the boundaries of cell. Epidermal growth factor receptor tyrosine kinase (EGFR-TKD) is a therapeutic target of breast cancer, prostate cancer and non-small lung cancer cells (NSLCC). Many drugs are approved by the FDA to mimic EGFR-TKD signalling in lung cancer cell lines. Erlotinib was the first-generation FDA-approved drug used against non-small lung carcinoma because of its high recovery rate among cancer patients, up to $70 \%$ in clinical trials. The binding of Erlotinib was found in both active and inactive types of EGFR-TKD 6 .

Erlotinib is a potent anti-cancer agent against EGFR-TKD, but causes mutations in many neoplastic cells after prolonged treatment and the TKI resistance is caused by the overexpression of the human epidermal receptor (HER2) ${ }^{7}$. The effectiveness of first and second-generation tyrosine kinase drugs shows promising results only in the initial stages, and thereafter, deletion of exon 19 accounts for $45 \%$ of the mutations in EGFR-TKD. Exon 18 and 20 mutations result loss of $\mathrm{P} 772, \mathrm{H} 773$, and the addition of $\mathrm{P}, \mathrm{C}$, and $\mathrm{Q}$ amino acids, respectively ${ }^{8-10}$. The double mutations occurred in EGFR-TKD by T790M/L858R (TMLR) and T790M/del $746-750$ (TMdel) is also known as point mutation $^{11,12}$

However, the efficacy of new drugs is limited because of resistance occurring from the second mutation (T790M) in EGFR-TKD. The gatekeeper amino acid Threonine 790 in the tyrosine kinase domain conferred binding specificity 
towards tyrosine kinase inhibitors (TKIs). This resistance blocks the kinase inhibitor's ability to bind with EGFRTKD $^{13}$. Overall, mutations by nonspecific drugs towards EGFR-TKD are a major concern in the present scenario. The resistance by marketed drugs, as well as their lethality, are major concerns in conventional therapeutics ${ }^{14,15}$. Therefore, the urge for novel effective therapeutic anticancer drugs is a remarkable challenge worthy of attention in cutting-edge technology ${ }^{13,16}$. Previous research has demonstrated that quinazolinones are EGFR-TKD inhibitors, and it has been proposed that the development of new heterocyclic thiazolo-[2,3-b] quinazolinones could be used as an EGFR-TKD inhibitor In this study we aimed to explore the molecular interactions via molecular docking simulations, molecular dynamic simulations and free energy binding (FEB) of newly synthesised heterocyclic thiazolo-[2,3-b] quinazolinones library against wild type EGFR-TKD (wtEGFR) and EGFR-TKD mutant "TMLR".

\section{Material And Methods}

\section{System preparation and protein-ligand docking}

The synthesised congeners 5aa-5aq, 5ba-5bq and reference ligands of wtEGFR-TKD (AQ44) and EGFR-TKD mutant "TMLR" (4ZQ) were sketched by using Avogadro software ${ }^{17}$. All molecules were optimised via the steepest decent method. These ligand molecules were reminimized in MOE $09^{18,19}$ by using the Molecular Merck force field (MMFF94). The wild type EGFR-TKD is bound with erlotinib (AQ44) PDB ID 1M17 and (1R)-1-\{6-(\{2-[(3R,4S)-3-fluoro4-methoxypiperidin-1-yl] pyrimidin-4-yl\} amino) [(2S)] -1-[(2S)]1,1,1-trifluoropropan-2-yl]-1 H-imidazo [4,5-c] Pyridin-2yl\} ethanol (4ZQ) bound with EGFR-TKD mutant "TMLR" PDB ID 5CAS were obtained from the RCSB database, www.rscb.org ${ }^{20,11}$. The resolution of wtEGFR-TKD $1 \mathrm{M} 17$ is $2.60 \AA$ and total weight is $38.27 \mathrm{kDa}$ and EGFR-TKD mutant "TMLR" 5CAS is $2.10 \AA$ and total weight of protein is $38.13 \mathrm{kDa}$. Both protein structures were minimized by using Gromacs 5.1.1 for 10ps and dumped in PDB format after minimizations. The binding site of both proteins is defined by using Argus Lab software ${ }^{21}$. The reference ligand (AQ44) of $1 \mathrm{M} 17$ was selected and made into a ligand group. The ligand group is selected and module "make a binding group" is used to identify the amino acids present in the binding site of the EGFR-TKD (1M17). A similar procedure was applied to the EGFR-TKD mutant "TMLR" and the amino acids present in the binding site were identified. These amino acids will be selected in a grid box for molecular docking simulations. AutoDock $4^{22}$ is used for estimation of the inhibition constant, ligand efficiency, and binding energy. The proteins were prepared by deleting water molecules, incorporating polar hydrogens, adding a Kollam charge. The library of thiazolo-[2,3-b] quinazolinones was prepared by selecting a torsion tree, adjusting the number of torsions, and saving them in pdbqt format. The grid dimensions of $1 \mathrm{M} 17$ at centre are $62 \times 46 \times 58(x, y$, $z$ ) and grid spacing resolutions are adjusted at $0.358, \AA$, grid box centre $x=25.517, y=0.052$ and $z=54.719$.

The 5CAS grid dimensions at the centre are $54 \times 42 \times 70(x, y, z)$, and the grid spacing resolution is set to 0.358 , with grid box centre $x=-51.385, y=1.059$, and $z=-24.769$. All the amino acids present in the binding site are covered in a grid box and form a binding site for molecular docking. The Autogrid module of AutoDock4 is used to build maps of atoms present in a ligand. The parameter search genetic algorithm is selected. 10 docking poses are opted for every ligand against both proteins (wtEGFR-TKD and EGFR-TKD mutant "TMLR"). The Lamarckian GA module is used to generate final docking poses by Autodock4. Each docking conformation is analysed by free energy binding scores. These scores are calculated on the basis of the sum of the distances between atom pairs.

$E=\sum$ epair $(d)$

(1).

where, epair(d)=w1*Gauss1(d)+w2*Gauss2(d)+w3*Repulsion(d)+w4*Hydrophobic(d)+w5*HBond(d) 
The molecular dynamics simulations were carried out using Gromacs 5.1.1 23,24. Ligand topologies are generated by using https://cgenff.umaryland.edu ${ }^{25-27}$. The charmm36 force field and recommended water model TIP 3-point are used ${ }^{28,29}$. Both Ligand and protein topologies files are incorporated in a single gromacs file. Gradually, this complex file is placed in the simulation box, which is $1 \mathrm{~nm}$ apart from the walls of the periodic boundary and solvates the box ${ }^{30}$. The prepared system is neutralized by using $\mathrm{Na}^{+}$and $\mathrm{Cl}$. The Steepest decent method is used for energy minimization. The temperature was kept at 300K and the pressure at 1 bar. Berendsen coupling of pressure and V-rescale coupling temperature is used. The Particle Mesh Ewald (PME) algorithm is used. The shortrange distance cut-off and coulomb cut-off are kept at $1.2 \mathrm{~nm}$. The LINICS algorithm is used to constrain the bond length ${ }^{31}$ for $2 \mathrm{fs}$. Both NVT and NPT methods are used to equilibrate the system for $100 \mathrm{ps}^{32} .8$ production runs of $20 \mathrm{~ns}$ simulations are carried out by applying a time step of $2 \mathrm{fs}$.

\section{Free Energy Calculations:}

Free energy binding is calculated by using g_mmpbsa. Three input trajectory files (trr or xtc), a topology parameter file (tpr), an index file (ndx), and an mdp python script for mmpbsa calculations are obtained from https://github.com/RashmiKumari/g_mmpbsa. The above-mentioned input files are obtained after completion of molecular dynamic simulation ${ }^{33}$.

The binding free energy of wtEGFR-TKD and EGFR-TKD mutant "TMLR" is calculated by the given equation.

$\Delta \mathrm{Gbinding}=\mathrm{G}_{\text {complex }}-\left(\mathrm{G}_{\text {protein }}+\mathrm{G}_{\text {ligand }}\right)(\mathrm{II})$.

$\mathrm{G}_{\text {complex }}$ denotes the total free energy of the protein-ligand complex, whereas the isolated total free energy of protein and ligand are represented as $G_{\text {protein }}$ and $G_{\text {ligand }}$ in the above given equation.

The free energy of an individual entity is summed up by the equation given below:

$\mathrm{GX}=\mathrm{E}_{\mathrm{MM}}-\mathrm{TS}+\mathrm{G}_{\text {solvation }}$ (III).

The protein ligand complex is denoted by $\mathrm{X}$, and $\mathrm{E}_{\mathrm{MM}}$ denotes an average molecular mechanics potential energy in vacuum, as calculated by equation (IV).

$\mathrm{G}_{\text {solvation }}$ denotes the free energy of solvation, and TS denotes the entropic free energy contribution in vacuum, while $\mathrm{T}$ denotes temperature and S entropy.

$E_{M M}=E_{\text {bonded }}+E_{\text {nonbonded }}=E_{\text {bonded }+} E_{v d v}+E_{\text {elec }}(I V)$.

$\mathrm{E}_{\text {bonded }}$ represents the collective interactions of the dihedral, angle, and bond. whereas the nonbonded interactions consist of van der Waals $\left(\mathrm{E}_{\mathrm{vdW}}\right)$ and electrostatic (Eelec) interactions.

Furthermore, Free Energy of Solvation, Polar Solvation Energy, Non-polar Solvation Energy, SASA-Only and polar models are calculated. The decomposed contributions of the free energy binding of each amino acid are calculated from $\Delta \mathrm{E}_{\mathrm{MM}}, \Delta \mathrm{G}_{\text {polar, }}$ and $\Delta \mathrm{G}_{\text {nonpolar. }}$

Assessment of ADMET properties of thiazolo-[2,3-b] quinazolinone derivatives and reference bound molecules with wild type EGFR-TKD (AQ44) and EGFR-TKD mutant “TMLR” (4ZQ). 
The $p k C S M^{34}$ Molsoft L.L.C ${ }^{35}$ and SwissADME ${ }^{36}$ servers are used to calculate toxicity, druglikeness and pharmacokinetic properties of thiazolo-[2,3-b] quinazolinones and reference molecules of wild type EGFR-TKD (AQ44) and EGFR-TKD mutant "TMLR" (4ZQ). Overall, these servers predict the toxic fragments of molecular structure in a very robust manner. The scrutiny of the molecular scaffolds is calculated on the basis of a weighed drug index and pharmacokinetic properties that are very useful in drug discovery. First, universal SMILES are obtained from Open Babel ${ }^{37}$ then these SMILES are used as inputs to obtain druglikeness, toxicity, and pharmacokinetics.

\section{Results And Discussions:}

Generally, many kinase inhibitors bind directly with the EGFR-TKD and inhibit the function of p-glycoprotein in malignant cells ${ }^{38}$. The synthesized quinazolinone based rhodamines are potent anti-cancerous agents ${ }^{39}$. Imidazole's and benzimidazoles are the functional precursors of polyazaheterocycles that are synthesized with the aid of microwave-mediated multi domino reactions MDRs ${ }^{40,41}$. New thiazolo [2,3-b] quinazolinone derivatives 5ab, 5aq and 5bq (Figure 1) are synthesized by microwave-assisted organic synthesis (MAOS) and multi-domino reaction (MDR). The thiazolo [2,3-b] quinazolinones have ADMET properties. Both the MDR and MAOS methods are cost effective, efficient and have remarkable structural attributes for anticancer activity. These molecular structures contain pyrimidine, cyclohexanone, and thiazole, which have a broader range of pharmaceutical properties. Quinazolinone has been synthesized by the substitution of phenyl and methyl groups over the benzene ring, which shows cytotoxic activity ${ }^{39}$. The thiazolo [2,3-b] quinazolinone derivatives share structural identity with the reference ligands of wtEGFR-TKD and EGFR-TKD mutant "TMLR", which contain a substituted pyrimidine moiety showing anticancer activities.

\subsection{Molecular docking simulations:}

The wild type EGFR-TKD and EGFR-TKD mutant "TMLR" are selected for in silico investigations. The PDB ID of wild type is noted as $1 \mathrm{M} 17$ and EGFR-TKD mutant "TMLR" as 5CAS. The thiazolo-[2,3-b] quinazolinone library is synthesized, and ADMET studies show that the derivatives 5ab, 5aq, and 5bq are not toxic as per predictions (Table SI-SIII). Furthermore, the comparative in silico investigations of three selected thiazolo-[2,3-b] quinazolinone derivatives (5ab, 5aq, 5bq) and reference ligands (AQ44 and 4ZQ) of wild type and EGFR-TKD mutant "TMLR" were done. The molecular docking interactions of thiazolo-[2,3-b] quinazolinones and the reference ligand erlotinib (AQ44) with the wtEGFR-TKD binding site reveal interactions with GLN 767, MET 769, THR 830, and ASN 831 in the binding site of wtEGFR-TKD, erlotinib (AQ44) interacted with amino acid MET 769 at position 3. The phenyl group of $5 \mathrm{ab}$ showed interactions with the N2 of Met769 and the 0 atom of GLN767 and N of ASP831 with the thiazolo[2,3-b] quinazolinone scaffold. The 5aq derivative interacted with the N of ASP831, the OG1 of THR830, the N2 of MET769, and the 0 atom of GLN767. The van der Walls interaction occurs within a $3 \AA$ range (Figure 2). The 5bq derivative of thiazolo-[2,3-b] quinazolinone exhibits van der walls interactions by the functional groups at the position 3 with MET769. Erlotinib drug molecule showed interaction with MET 769 by H-bond at conserved hinge region with the $\mathrm{N} 1$ atom of the quinazoline ring as reported earlier by Stamos et al., 2020. However, the similar interactions were found in synthesized ligands and erlotinib, hence these thiazolo-[2,3-b] quinazolinones derivatives may also be the potent inhibitor of wtEGFR-TKD. Molecular docking interactions exploited by thiazolo-[2,3-b] quinazolinones (5ab, 5aq and 5bq) and EGFR-TKD mutant "TMLR" reference (4ZQ) are analysed. Amino acids Lys745, Gln762, Asn842, Asp855, Gln791, Met793, GIn791 and Thr854 exhibit interactions with ligand moieties. 
Also, amino acid Lys745 interacts with thiazolo [2,3-b] quinazolinone derivatives (5ab, 5aq, and 5bq) and reference ligand (4ZQ) via an aryl substituted functional group with a binding cavity. Among these three derivatives, 5ab exhibits interactions with the N atom of LYS745 and ASP 855 (Figure 3). Similar interactions are found in 5 ad and $5 \mathrm{bq}$ and the interactions are showed with ASP855 by the $\mathrm{OH}$ group flanked by cyclohexanone and pyrimidine rings at position 9a. The reference (4ZQ) ligand of the EGFR-TKD mutant "TMLR" showed interactions with the $\mathrm{N}$ atoms MET793. Besides these interactions, THR854 also interacts with the $\mathrm{N}$ atom of the pyrimidine ring during molecular docking simulations. The free binding energy, ligand efficiency, and inhibition constant of thiazolo [2,3-b] quinazolinone derivatives against wtEGFR-TKD are given in (Table I) and for EGFR-TKD mutant "TMLR" in (Table II). The mean energy of binding is obtained in triplicates. The highest negative binding energy is found in $5 \mathrm{aq}=-11.97$ $\mathrm{kcal} / \mathrm{mol}$.

Table I. Molecular docking results of thiazolo-[2,3-b] quinazolinone derivatives and reference ligand (AQ44) of 1M17 with wtEGFR-TKD by using ADT in triplicates.

\begin{tabular}{|c|c|c|c|c|c|}
\hline SI No. & Ligand & Inhibition Constant (Ki) & Free Energy Binding (Kcal/mol) & Ligand efficiency & which is \\
\hline 1 & $5 a b$ & $444.37 \pm 8.353 \mathrm{nM}$ & $-8.67 \pm 0.0133$ & $-0.29 \pm 0.00$ & \\
\hline 2 & $5 \mathrm{aq}$ & $1.65 \pm 0.029 \mathrm{nM}$ & $-11.98 \pm 0.0115$ & $-0.36 \pm 0.00$ & oved \\
\hline 3 & $5 b q$ & $879.62 \pm 6.725 \mathrm{nM}$ & $-8.26 \pm 0.0033$ & $-0.27 \pm 0.00$ & \\
\hline 4 & AQ44 & $3.07 \pm 0.717 \mu \mathrm{M}$ & $-7.54 \pm 0.1411$ & $-0.26 \pm 0.005$ & mean \\
\hline
\end{tabular}
energy of

binding of thiazolo [2,3-b] quinazolinone derivatives is comparatively similar to that of reference ligand (4ZQ) towards the EGFR-TKD mutant "TMLR". The results of molecular docking revealed that the selected thiazolo [2,3-b] quinazolinone derivatives may be the inhibitors of both wtEGFR-TKD and EGFR-TKD mutant "TMLR". Further molecular docking is linked with molecular dynamic simulations and MM-PBSA calculations for conformations of ligand movement, root mean square fluctuations, and free energy binding at an atomistic level against both wild type and EGFR-TKD mutant "TMLR".

Table II. Molecular docking results of thiazolo-[2,3-b] quinazolinone derivatives and reference ligand (4ZQ) with EGFR-TKD mutant "TMLR” by using ADT in triplicates.

\begin{tabular}{|lllll|}
\hline SI No. & Ligand & Inhibition Constant (Ki) & Free Energy Binding (Kcal/mol) & Ligand efficiency \\
\hline $\mathbf{1}$ & $5 \mathrm{ab}$ & $838.42 \pm 5.84 \mathrm{nM}$ & $-8.29 \pm 0.006$ & \\
\hline $\mathbf{2}$ & $5 \mathrm{aq}$ & $765.47 \pm 118.5 \mathrm{nM}$ & $-8.36 \pm 0.101$ & $-0.28 \pm 0.006$ \\
\hline $\mathbf{3}$ & $5 \mathrm{bq}$ & $1.52 \pm 0.23 \mu \mathrm{M}$ & $-7.95 \pm 0.088$ & $-0.25 \pm 0.003$ \\
\hline $\mathbf{4}$ & $4 \mathrm{ZQ}$ & $201.9 \pm 6.98 \mathrm{nM}$ & $-9.13 \pm 0.018$ & $-0.27 \pm 0.003$ \\
\hline
\end{tabular}

\subsection{D visualization of protein-ligand interactions:}


After completion of molecular docking simulations, protein-ligand complexes were introduced to obtain interactions exploited by thiazolo-[2,3-b] quinazolinone and reference ligands (AQ44, 4ZQ) with the catalytic site of wild and EGFR-TKD mutant "TMLR" by using MOE v200918-19. All the molecular interactions exploited are presented in Figure 2 and 3.

\subsection{Molecular dynamic simulations:}

MD simulations are a powerful tool for discovering new inhibitors and investigating drug resistance mechanisms ${ }^{42,43}$. Molecular dynamic simulations of thiazolo [2,3-b] quinazolinone derivatives (5ab, 5aq, and 5bq), reference ligands of both wtEGFR-TKD and EGFR-TKD mutant "TMLR" were done. The root means square deviations of ligand movement, ligand conformations, and receptor Ca were evaluated. Further root means square fluctuations, solvent accessible surface area, radius of gyration, and number of hydrogen bonds formed were analyzed. The comparative analysis was done with wtEGFR-TKD and EGFR-TKD mutant "TMLR". The reference ligands of both wtEGFR-TKD and EGFR-TKD mutant "TMLR" were compared with thiazolo [2,3-b] quinazolinones on the basis of stability with the catalytic sites of receptor protein tyrosine kinases.

\subsubsection{Evaluation of RMSD of Ligand movement, Ligand conformations and receptor Ca during simulations with wtEGFR-TKD.}

The RMSD of thiazolo [2,3-b] quinazolinone derivatives 5ab, 5aq, 5bq and references ligand (AQ44) are analysed with $1 \mathrm{M} 17$ wtEGFR-TKD during simulations. (Figure 4a). The RMSD of 5ab when superimposed to $1 \mathrm{M} 17$ is $0.25-$ $0.35 \mathrm{~nm}$ in an aqueous medium during simulations. Similarly, the ligand movement of the 5aq-1M17 complex is between $0.08-0.34 \mathrm{~nm}$ for $20 \mathrm{~ns}$ of the simulation period. The RMSD $5 \mathrm{bq}-1 \mathrm{M} 17$ complex is $0.05-0.3 \mathrm{~nm}$ throughout the simulation period. Furthermore, the RMSD trajectory of the reference ligand (AQ44) of wtEGFR-TKD is $0.08-0.28 \mathrm{~nm}$.

The ligand conformations When 5ab, 5aq, 5bq, and reference ligand (AQ44) are superimposed into the respective ligand, the RMSD is calculated (Figure $4 b$ ). The RMSD of $5 a b$ is $0.04-0.13 \mathrm{~nm}$, $5 \mathrm{aq}$ is $0.03-0.12 \mathrm{~nm}$, and $5 \mathrm{bq}$ is $0.03-0.1 \mathrm{~nm}$ during simulations, and the RMSD of reference ligand (AQ44) is between 0.05 to $0.2 \mathrm{~nm}$ during simulation period. The $1 \mathrm{M} 17$ receptor $\mathrm{Ca}$ RMSD is analysed when simulated with $5 \mathrm{ab}, 5 \mathrm{aq}, 5 \mathrm{bq}$ and AQ44 (Figure 4c). The RMSD Ca of the complexes 5ab-1M17, 5aq-1M17, and 5bq-1M17 are $0.1-0.38 \mathrm{~nm}, 0.1-0.35 \mathrm{~nm}$, and $0.1-0.4 \mathrm{~nm}$, respectively. It is $0.15-0.38 \mathrm{~nm}$ during the simulation period when simulated with reference ligand (AQ44).

\subsubsection{Root Mean Square Fluctuations of wtEGFR-TKD.}

The flexibility of amino acids is determined by root mean square fluctuations and is corroborated that the flexibility of amino acids is higher in the EGFR-TKD mutant "TMLR" than in wtEGFR-TKD ${ }^{45,46}$. The RMSF of wtEGFR-TKD is analyzed when simulated with different derivatives of thiazolo-[2,3-b] quinazolinones and AQ44 (Figure 4d). The RMSF of wtEGFR-TKD with $5 \mathrm{ab}$ is $0.1-0.6 \mathrm{~nm}$ at the $\mathrm{N}$ terminus, the $\mathrm{C}$ terminus RMSF is $0.1-0.9 \mathrm{~nm}$, whereas the remaining amino acid fluctuations are in the range of $0.1-0.4 \mathrm{~nm}$. However, the RMSF of $1 \mathrm{M} 17$ when simulated with $5 \mathrm{aq}$ is $0.1-0.82 \mathrm{~nm}$ at the $\mathrm{N}$ terminus and the remaining amino acid fluctuations are between $0.1-0.38 \mathrm{~nm}$. The RMSF of wtEGFR-TKD when simulated with $5 \mathrm{bq}$ derivative is $0.1-1.25 \mathrm{~nm}$ at the $\mathrm{C}$ terminus, whereas the $\mathrm{N}$ terminus fluctuations are $0.1-0.6 \mathrm{~nm}$. The remaining amino acid fluctuations are between $0.1-0.4 \mathrm{~nm}$ during simulations. The RMSF of wtEGFR-TKD when simulated with reference AQ44 is approximately $1 \mathrm{~nm}$ at the $\mathrm{C}$ and $\mathrm{N}$ terminus, whereas the remaining amino acid RMSF is between $0.1-0.35 \mathrm{~nm}$. 


\subsubsection{Radius of gyration and solvent accessible surface area of wtEGFR-TKD.}

The variations in protein stability differ between wild type and EGFR-TKD mutant "TMLR". Wild type EGFR holds greater stability than mutated EGFR ${ }^{45}$. The compactness of receptor proteins ( $1 \mathrm{M} 17$ \& $5 \mathrm{CAS}$ ), motion among amino acids of wtEGFR-TKD and EGFR-TKD mutant "TMLR" are determined during simulations Higher compactness reflects low protein folding, and vice-versa, higher amino acid fluctuation results in less stability. Figure 4 e depicts the radius of gyration of wtEGFR-TKD during simulation with thiazolo-[2,3-b] quinazolinone derivatives and AQ44. The reflected Rg values of $1 \mathrm{M} 17$ with the 5ab derivative are between $1.97-2.05 \mathrm{~nm}$ and SASA values are between $150-170 \mathrm{~nm}^{2}$ (Figure 4f). When the $1 \mathrm{M} 17$ main chain was simulated with $5 \mathrm{aq}$, the Rg value was $1.95-2.03 \mathrm{~nm}$ and the SASA value was 151-167 nm2.The Rg values of $1 \mathrm{M} 17$ when simulated with $5 \mathrm{bq}$ are $1.95-2.05 \mathrm{~nm}$ and the SASA value is $152.5-168 \mathrm{~nm}^{2}$ during the simulation period. The Rg values of $1 \mathrm{M} 17$ main chain are $1.95-2.05 \mathrm{~nm}$ when simulated with AQ44 and the SASA values are $155-167 \mathrm{~nm}^{2}$ for the first 6

$\mathrm{ns}$ of the simulation period, then the motion of amino acids decreases and the SASA value is $152-165 \mathrm{~nm}^{2}$ for the remaining simulation period.

\subsubsection{Evaluation of RMSD of Ligand movement, Ligand conformations and receptor Ca during simulations with EGFR-TKD mutant “TMLR”.}

The RMSD of 5ab, 5aq, 5bq, and reference ligand 4ZQ with MT EGFR-TKD during simulations is analyzed (Figure 5a). The RMSD of the 5ab-5CAS complex for $10 \mathrm{~ns}$ of simulations is $0-0.75 \mathrm{~nm}$, then the value increases slightly for the next $3 \mathrm{~ns}$, which are within the range of $0.75-1.2 \mathrm{~nm}$. Furthermore, the RMSD value are within $0.75-1 \mathrm{~nm}$ from $13-20$ ns of simulation. Similarly, the RMSD of the 5aq-5CAS complex during simulations is within $0.2-0.6$ $\mathrm{nm}$ and the 5bq-5CAS complex shows RMSD of $0-0.5 \mathrm{~nm}$ for the first $3 \mathrm{~ns}$ and $0.4-0.5 \mathrm{~nm}$ for the rest of the simulation period. The RMSD of $4 Z \mathrm{Q}$ is $0.2-0.3 \mathrm{~nm}$ for the first $3.5 \mathrm{~ns}$, then the RMSD trajectory remains between $0.08-0.8 \mathrm{~nm}$ throughout the simulation period when simulated with $5 \mathrm{CAS}$.

The RMSDs of thiazolo-[2,3-b] quinazolinone derivatives and 4ZQ when superimposed to respective ligand are calculated and the RMSD of $5 \mathrm{ab}$ is $0.2-0.4 \mathrm{~nm}$, $5 \mathrm{aq}$ is $0.06-0.16 \mathrm{~nm}$, and $5 \mathrm{bq}$ is $0.04-0.15 \mathrm{~nm}$. However, the reference ligand RMSD value is within $0.05-0.16 \mathrm{~nm}$ during simulations (Figure $5 \mathrm{~b}$ ). The receptor Ca RMSD is analyzed (Figure 5c) when simulated with 5ab, 5aq, 5bq and reference 4ZQ. The Ca RMSD of the 5ab-5CAS complex is $0.2-0.4 \mathrm{~nm}$, 5aq-5CAS complex is within the range of $0.2-0.46 \mathrm{~nm}$, the $5 \mathrm{bq}-5 \mathrm{CAS}$ complex shows a RMSD of $0.15-0.35 \mathrm{~nm}$ and the 4ZQ-EGFR-TKD mutant "TMLR" complex value is $0.05-0.16 \mathrm{~nm}$ during the simulation period. According to the data, 5bq thiazolo-[2,3-b] quinazolinone derivatives are less stable with EGFRTKD mutant "TMLR" than wtEGFR-TKD and other derivatives. Both 5ab and 5aq showed maximum stability with both EGFR-TKDs during simulation in an aqueous medium.

\subsubsection{Root Mean Square Fluctuations of EGFR-TKD mutant "TMLR"}

The RMSF of EGFR-TKD mutant "TMLR" is analyzed during simulations with thiazolo-[2,3-b] quinazolinone derivatives and 4ZQ (Figure 5d). The EGFR-TKD mutant "TMLR" (5CAS) shows RMSF of $1 \mathrm{~nm}$ in the $\mathrm{C}$ terminus, whereas the rest of the amino acids show $0.09-0.4 \mathrm{~nm}$ fluctuations when simulated with the 5ab-5CAS complex. The RMSF of 5 CAS at the $\mathrm{N}$ terminus is $0.15-0.6 \mathrm{~nm}$, at the $C$ terminus it is $0.9 \mathrm{~nm}$ and the remaining amino acid RMSF is found within $0.15-0.25 \mathrm{~nm}$ when simulated with the 5aq-5CAS complex. Similarly, the fluctuations found in the $\mathrm{N}$ and $\mathrm{C}$ terminus lie within $0.02-0.8 \mathrm{~nm}$ and $0.002-1 \mathrm{~nm}$ respectively, and the remaining amino acids show 0.02 - $0.4 \mathrm{~nm}$ fluctuations when simulated with the 5bq-5CAS complex. The RMSF of the 4ZQ-5CAS complex 
is $0.07-1 \mathrm{~nm}$ at the $\mathrm{N}$ terminus, $0.07-0.4 \mathrm{~nm}$ at the $\mathrm{C}$ terminus, and the remaining amino acid fluctuations are $0.07-2.2 \mathrm{~nm}$. The present findings support previous results ${ }^{45,46}$. The flexibility of various amino acids in the EGFRTKD mutant "TMLR" is higher than in wtEGFR-TKD.

\subsubsection{Radius of gyration and solvent accessible surface area of EGFR-TKD mutant "TMLR”.}

The Rg and SASA values reflected during simulations of EGFR-TKD mutant "TMLR" (5CAS) are found in the range of $2-2.08 \mathrm{~nm}$ and the SASA value of the protein lies within $157-175 \mathrm{~nm} 2$ when simulated with the 5ab derivative. When simulated with $5 \mathrm{aq}$, the $\mathrm{Rg}$ of the main chain is found to be $2-2.12 \mathrm{~nm}$, and the SASA of the protein is 155$185 \mathrm{~nm}$ 2.The Rg values of the main chain and SASA of protein values are similar as in 5 aq when simulated with $5 \mathrm{bq}$ (Figure $5 \mathrm{e} \& \mathrm{f}$ ). When simulated with 4ZQ, the Rg value of the main chain of 5CAS is $1.99-2.05 \mathrm{~nm}$, and the SASA value of the protein is 157 - $174 \mathrm{~nm}$, which is comparable to other thiazolo-[2,3-b] quinazolinone derivatives. The radius of gyration analysis shows EGFR-TKD mutant "TMLR" has more variations in protein stability in comparison to wtEGFR-TKD. These findings agree with previously reported protein stability of wtEGFR-TKD and mutated EGFR-TKD ${ }^{46}$.

\subsubsection{Hydrogen bond analysis:}

The hydrogen bond module of gromacs is used to obtain the number of hydrogen bonds formed between protein and ligand. The hydrogen bond analysis among thiazolo [2,3-b] quinazolinone derivatives and reference ligands of wtEGFR-TKD and EGFR-TKD mutant "TMLR" during MD simulations has been determined. The hydrogen bond formed by thiazolo [2,3b] quinazolinone derivative 5ab with wtEGFR-TKD is 1, 5aq, 5bq derivative and reference ligand AQ44 formed 2 hydrogen bonds each (Figure 6a-d). EGFR-TKD mutant "TMLR" 5ab and 5aq derivative formed 4 hydrogen bonds, 5 bq derivative and reference ligand (4ZQ) formed 2 hydrogen bonds, which is higher than the wtEGFR-TKD complexes (Figure 7a-d). In both cases, hydrophobic, van der Walls, and hydrogen bonding play pivotal roles in binding with wtEGFR-TKD and EGFR-TKD mutant "TMLR". The hydrogen bonds formed during simulations validate the molecular docking results.

\subsection{Comprehensive analysis of free energy binding of thiazolo [2,3-b] quinazolinone with wtEGFR-TKD and EGFR- TKD mutant "TMLR".}

The negative free energy binding of both wild type EGFR-TKD and EGFR-TKD mutant "TMLR" with thiazolo-[2,3-b] quinazolinone derivatives and reference ligands is determined. The python code MmPbSaStat.py is used in the present study to compare the binding affinities of thiazolo-[2,3-b] quinazolinone derivatives with wtEGFR-TKD and EGFR-TKD mutant "TMLR". The bootstrap module of g_mmpbsa with APBS is used to calculate the average binding energy with SD/error. The energy generated is raised in the form of binding energy. Collectively, end point free binding energy is a sum of changes in electrostatic energy, van der Waals energy, SASA energy, and polar solvation energy. The low negative free binding energy of a molecule determines the potency towards the target.

\subsubsection{End point binding free energy of thiazolo-[2,3-b] quinazolinone derivatives and AQ44 with wtEGFR-TKD:}

Each derivative of thiazolo-[2,3-b] quinazolinone and AQ44 is selected for free binding energy calculations. The average binding free energy $\Delta$ Gbind of $5 \mathrm{ab}$ is $-40.503 \pm 0.435 \mathrm{~kJ} / \mathrm{mol}, 5 \mathrm{aq}=-51.551 \pm 0.409 \mathrm{~kJ} / \mathrm{mol}, 5 \mathrm{bq}$ $=-28.773 \pm 0.597 \mathrm{~kJ} / \mathrm{mol}$ and $\mathrm{AQ} 44=-76.378 \pm 0.355 \mathrm{~kJ} / \mathrm{mol}$ are calculated by bootstrap analysis of the g_mmpbsa module. This binding free energy determines that $\mathrm{AQ} 44$ has a much higher negative binding energy than thiazolo- 
[2,3-b] quinazolinone derivatives (Table III, Figure 8), but 5bq also has a high negative free binding energy and they are parallel with $\mathrm{AQ} 44$ reference ligand.

3.4.2. End point binding free energy of thiazolo-[2,3-b] quinazolinone derivatives and reference ligand $4 Z \mathrm{Z}$ with $5 \mathrm{bq}-$ EGFR-TKD MUTANT "TMLR”:

Similarly, the free energy of thiazolo-[2,3-b] quinazolinone derivatives and 4ZQ with EGFR-TKD mutant "TMLR" is calculated from 2000 snapshots. The average binding free energy of $5 \mathrm{ab}$ is- $47.064 \pm 0.763 \mathrm{~kJ} / \mathrm{mol}, 5 \mathrm{aq}$ $=-63.723 \pm 0.419 \mathrm{~kJ} / \mathrm{mol}, 5 \mathrm{bq}=-41.212 \pm 0.834 \mathrm{~kJ} / \mathrm{mol}$ and $4 Z \mathrm{Q}=-71.938 \pm 0.367 \mathrm{~kJ} / \mathrm{mol}$. 4ZQ shows a higher negative binding energy than other derivatives of thiazolo [2,3-b] quinazolinone (Table IV, Figure 8).

The 5ab, 5aq, 5bq thiazolo-[2,3-b] quinazolinone derivatives and reference ligands with wtEGFR-TKD and EGFR-TKD mutant "TMLR" reveal $5 \mathrm{ab}$ show $\triangle$ Gbind $=-7.0 \mathrm{~kJ} / \mathrm{mol}$ lower against EGFR-TKD mutant "TMLR" than wtEGFR-TKD, besides 5 ab derivative the 5 aq thiazole-[2,3-b] quinazolinone derivative show $\Delta$ Gbind lower $=-12.0 \mathrm{~kJ} / \mathrm{mol}$ approximately against EGFR-TKD mutant "TMLR" than wtEGFR-TKD. The 4ZQ show $-8.26 \mathrm{~kJ} / \mathrm{mol}$ lower free energy binding than derivative 5aq, hence this MM-PBSA calculations revealed the higher negative free energy binding of thiazolo-[2,3-b] quinazolinone derivatives against EGFR-TKD mutant "TMLR" not more than 4ZQ. The estimated $\triangle G$ bind value of thiazole- [2,3-b] quinazolinone derivatives against wtEGFR-TKD complexes and EGFR-TKD mutant "TMLR" complexes concludes thiazole [2,3-b]- quinazolinone derivatives may have higher inhibitory potency towards EGFR-TKD mutant "TMLR" than wtEGFR-TKD. Lower negative free energy values were identified against EGFR-TKD mutant "TMLR" complexes than wtEGFR-TKD after completion of the MMPBSA calculations.

Table III. Binding energy components of thiazolo [2,3-b] quinazolinone derivatives -wtEGFR-TKD complexes and reference ligand complex obtained by using g_mmpbsa module.

\begin{tabular}{|lllllll|}
\hline $\begin{array}{l}\text { SI } \\
\text { No. }\end{array}$ & Ligand & $\Delta \mathrm{E}_{\mathrm{VdW}}$ & $\Delta \mathrm{E}_{\mathrm{Elec}}$ & $\Delta \mathrm{G}_{\text {polar }}$ & SASA & $\Delta \mathrm{G}_{\text {binding }}$ \\
\hline 1. & $5 \mathrm{ab}$ & $-116.979 \pm 0.370$ & $-15.912 \pm 0.346$ & $108.342 \pm 0.708$ & $-15.950 \pm 0.040$ & $-40.503 \pm 0.435$ \\
\hline 2. & $5 \mathrm{aq}$ & $-138.465 \pm 0.287$ & $-34.012 \pm 0.334$ & $138.948 \pm 0.741$ & $-18.012 \pm 0.031$ & $-51.551 \pm 0.409$ \\
\hline 3. & $5 \mathrm{bq}$ & $-128.029 \pm 1.187$ & $-57.300 \pm 0.729$ & $173.040 \pm 01.617$ & $-16.480 \pm 0.512$ & $-28.773 \pm 0.597$ \\
\hline 4. & $\mathrm{AQ} 44$ & $-174.685 \pm 0.466$ & $-45.909 \pm 0.258$ & $164.710 \pm 0.565$ & $-20.495 \pm 0.052$ & $-76.378 \pm 0.355$ \\
\hline
\end{tabular}

Table IV. Binding energy components of thiazolo [2,3-b] quinazolinone derivatives -EGFR-TKD mutant "TMLR" Complexes and reference ligand complex obtained by using g_mmpbsa module.

\begin{tabular}{|lllllll|}
\hline $\begin{array}{l}\text { SI } \\
\text { No. }\end{array}$ & Ligand & $\Delta \mathrm{E}_{\mathrm{VdW}}$ & $\Delta \mathrm{E}_{\mathrm{Elec}}$ & $\Delta \mathrm{G}_{\text {polar }}$ & \multicolumn{1}{l}{$\mathrm{SASA}$} & $\Delta \mathrm{G}_{\text {binding }}$ \\
\hline 1. & $5 \mathrm{ab}$ & $-85.251 \pm 1.009$ & $-26.104 \pm 0.516$ & $75.405 \pm 1.165$ & $-11.110 \pm 0.130$ & $-47.064 \pm 0.763$ \\
\hline 2. & $5 \mathrm{aq}$ & $-148.244 \pm 0.343$ & $-14.831 \pm 0.300$ & $117.929 \pm 0.491$ & $-18.574 \pm 0.036$ & $-63.723 \pm 0.419$ \\
\hline 3. & $5 \mathrm{bq}$ & $-51.842 \pm 0.921$ & $-35.394 \pm 0.729$ & $53.073 \pm 1.284$ & $-7.064 \pm 0.123$ & $-41.212 \pm 0.834$ \\
\hline 4. & $4 \mathrm{ZQ}$ & $-177.057 \pm 0.320$ & $-61.126 \pm 0.315$ & $187.366 \pm 0.678$ & $-21.115 \pm 0.027$ & $-71.938 \pm 0.367$ \\
\hline
\end{tabular}


All thiazolo-[2,3-b] quinazolinone derivatives (5ab, 5aq, and 5bq), AQ44, and 4ZQ against wtEGFR-TKD and EGFRTKD mutant "TMLR" are selected for the contribution of free energy decomposition, which is calculated by using the MmPbSaDecomp.py python script. The decomposition of contribution energy of each amino acid during free energy calculations is determined. Residues of wtEGFR-TKD and EGFR-TKD mutant "TMLR" contribute to free energy decomposition. The decomposition free energy contribution of the wtEGFR-TKD-5ab complex is found in amino acids, Leu694 $=-2.1811$, Val720 $=-5.7595$, lle720 $=-2.0354$ and Leu820 $=-4.3941$. In the 5aq-wtEGFR-TKD complex, free energy contributions are found in amino acids. Lys693 $=-4.8364$, Val720 $=-4.5307$ and Leu768 = -2.5720 amino acids. 5bq-wtEGFR-TKD complex free energy contributions are found in Leu694 = -6.0328, Val702 = -5.6625 , Leu768 $=-2.8350$, Cys773 $=-2.4046$ and Leu820 $=-5.0634$ amino acids. In addition, the AQ44-wtEGFR-TKD contribution to bindings is found inLeu694 =-6.2639, Val702 =-4.8866, Ala719 =-2.7918, Met742 =-2.6695, Thr766 $=-3.4538$, Leu768 =-4.4472, and Met769 =-1.2950 amino acids (Figure: 9 left panel).

This binding energy contribution analysis determined that Val702 is the common amino acid that participates in all complexes of wtEGFR-TKD in energy decomposition. Also, the decomposition energy of the 5ab-EGFR-TKD mutant "TMLR" complex is found among the amino acids Glu and Asp, which frequently participated in the contribution of free energy decomposition over 2000 frames. Glu values range from -8.9449 to- $21.9148 \mathrm{~kJ} / \mathrm{mol}$ and Asp decomposition of energy binding values is -11.5561 to $-19.443 \mathrm{~kJ} / \mathrm{mol}$. Also, Phe723 =-8.4733 and Pro1020 $=-10.3223 \mathrm{~kJ} / \mathrm{mol}$ are found during energy decompositions. The contributions of energy decomposition in the 5aqEGFR-TKD mutant "TMLR" complex are found in the amino acids Leu718 = -3.8968, Phe723 = -8.7553, Ala743 = -2.0249 , Met790 = -3.3889, Leu792 = -2.2623, Cys797 = -4.5374, and Leu844 =-5.4694 kJ/mol. The energy decomposition by 5bq-EGFR-TKD mutant "TMLR" complex is found frequently in Glu and Asp. Glu ranges from -7.5618 to $-15.9943 \mathrm{~kJ} / \mathrm{mol}$ and Asp -9.2642 to $-14.1010 \mathrm{~kJ} / \mathrm{mol}$. Besides these two amino acids, other amino acids, Phe723 = -4.6643 and Pro1019 $=-8.3479 \mathrm{~kJ} / \mathrm{mol}$, also participate in free energy decompositions. 4ZQ-EGFRTKD mutant "TMLR" complex energy decomposition is found in amino acids Leu718 = -4.8575, Phe723 = -4.6592, Met790 $=-5.4375$, Leu792 $=-4.0527$, Met793 $=-3.5920$ and Thr854 = -2.8694 kJ/mol (Figure: 9 right panel). Contribution free energy decomposition leads to the conclusion that the 5aq derivative of thiazolo-[2,3-b] quinazolinone has many similarities with the reference ligand (4ZQ). Average negative free energy binding calculations help to find the most potent molecule against wtEGFR-TKD and TMLR-EGFR-TKD therapeutic targets. The contribution of wtEGFR-TKD and "TMLR" EGFR-TKD to free energy decomposition with thiazolo-[2,3-b] quinazolinone derivatives (5ab, 5aq, 5bq), and reference ligand AQ44, 4ZQ complexes is represented as new cartoon graphic annotations with a free energy binding colour scale (Figure 10).

Despite the free energy contribution energy by amino acids of wild type and mutated type tyrosine kinases during calculations, the selected ligands also contribute towards free energy decomposition. The reference ligand AQ44 shows lower negative free energy contributions than $5 \mathrm{ab}$, 5aq, and 5bq, but 5 aq derivative shows a slight difference of- $4 \mathrm{~kJ} / \mathrm{mol}$ than AQ44. In the case of the EGFR-TKD mutant "TMLR", the 5aq derivative shows higher negative decomposition than the reference ligand $4 Z Q$. These conclusive results suggest that $5 \mathrm{bq}$, a derivative of thiazolo[2,3-b] quinazolinone, may also be a potent anti-cancer agent and may inhibit the functioning of both wild and EGFR-TKD mutant "TMLR".

\subsection{ADMET predictions:}


The pkCSM, SWISSadme, and Molsoft L.L.C. were used to investigate the toxicity, druglikeness of thiazolo [2,3-b] quinazolinones and reference ligands of both wtEGFR-TKD and MT EGFR-TKD. The only three 5ab,5aq and 5bq are found safe but reference ligands AQ44 and 4ZQ are inhibitors of HerG-II and show hepatotoxicity as per predictions. All the molecules taken in this study did not show AMES toxicity. Furthermore, none of the molecules violated the Lipski rule of 5 . The druglikeness index of $5 a b=0.66,5 a q=0.37,5 b q=0.62,4 Z Q=0.79$ and $A Q 44=$ 0.90. Higher druglikeness was reported in AQ44 and 4ZQ because they hold toxic fragments as per predictions. Thereafter, the ADMET analysis suggests toxic scaffolds have higher druglikeness than non-toxic drug candidates (Table SI-SIII). Experimental findings supported predicted results that erlotinib (AQ44) is a hepatoxic molecule ${ }^{46}$. We present non-toxic thiazolo-[2,3-b] quinazolinone derivatives synthesised using Insilico methods, which will be further evaluated by in vivo methods.

\section{Conclusion}

The goal of this research is to find the best inhibitor from thiazolo-[2,3-b] quinazolinone derivatives against wtEGFRTKD and EGFR-TKD mutants "TMLR". Preliminary research and deep machine learning data suggest that thiazolo$[2,3-b]$ quinazolinone derivatives could be the next generation anti-cancer drugs. Widely designed EGFR-TKD inhibitors were studied, and some of them are available on the market. These inhibitors have certain complications, and their specificity towards a target is still not known. We therefore introduced two chemotherapeutic targets (wtEGFR-TKD and EGFR-TKD mutant "TMLR") as they are present in a wide range of cancer cells. The stability and binding energy of thiazolo-[2,3-b] quinazolinone derivatives towards both wtEGFR-TKD and EGFR-TKD mutant "TMLR" are calculated by molecular dynamics simulation and MMPBSA ( $g$-mmpbsa) methods. According to MD simulations, the thiazolo-[2,3-b] quinazolinone $5 \mathrm{ab}, 5 \mathrm{aq}$, and $5 \mathrm{bq}$ derivatives are stable in the binding cavity of wtEGFR-TKD and EGFR-TKD mutant "TMLR". The free energy binding of 5ab and 5aq was much lower than that of 5 bq against wtEGFR-TKD, whereas 5aq showed the lowest negative free energy binding against the EGFR-TKD mutant "TMLR", which was investigated by the MM-PBSA method. According to predictions, thiazolo-[2,3-b] quinazolinone has ADMET properties and is non-toxic, but both reference ligands AQ44 and 4ZQ are hepatotoxic and are inhibitors of the human Ether-go-to-go gene (HerG-II). Furthermore, we conclude that the selected lead candidates of thiazolo-[2,3-b] quinazolinone derivatives may have EGFR-TKD inhibitory potency. This study will help to design the most potent inhibitor against wtEGFR-TKD and EGFR-TKD mutant "TMLR". Also, these molecular scaffolds will be taken to In vitro and In vivo levels for future study of interest.

\section{Data And Software Availability:}

The data mentioned in this article will be available upon the author's request. Further, MOE-09 was used and acknowledged, Chem Draw 8.0 was used for sketching the initials, some open-source agreement software such as Open Babel (http://openbabel.org/.) was used for ligand preparations, and VMD accessed at https://www.ks.uiuc.edu/Research/vmd/molecular visualisation programme was used for visualization, displaying, animating, and 3D diagrams. AutoDock 4 was used for molecular docking simulations: http://autodock.scripps.edu/. Avogadro http://www.avogadro.cc/ were used as molecule editors and visualizers. The MolSoft L.L.C www.molsoft.com/mprop/, SWISSadme, http://www.swissadme.ch/, and pkCSM, http://biosig.unimelb.edu/, were used to study the ligand toxicities and pharmacokinetic properties of molecular scaffolds selected in this study. Molecular dynamics were carried out by using gromacs $5.1 .1 \mathrm{https} / / /$ gromacs.org. Free software can either be redistributed or modified under the GNU Lesser Public License Agreement as provided by free software publication. CGenFF was used to generate topologies at https://cgenff.umaryland.edu/. Free

Page $12 / 24$ 
energy calculations were carried out by using gromacs 5.1.1, incorporated with APBS versions 1.2.x, 1.3.x, and all input files are available at https://github.com/RashmiKumari/g_mmpbsa. Xmgrace is freely available at https://ubuntuforums.org/showthread.php?t=442322. These were used to plot the trajectories obtained from molecular dynamics simulations. The data presented here were not obtained from any published literature and will not violate any future scientific publishing policy terms and conditions.

\section{Declarations}

\section{Authors Contribution:}

SAM carry in silico investigations, Molecular calculations at atomistic level and MM-PBSA calculations by using gromacs 5.1.1. SAM prepared the Draft, SAM and BN edited and finalized the Manuscript.

\section{Funding Source:}

No funding source committed.

\section{Conflict of Interest:}

The authors declare not conflict of interest

\section{Acknowledgement:}

Both authors acknowledge Dr. Ibrahim Khalifa Benha University, Egypt, for their kind gift of MOE09.

\section{References}

1. Siegel, RL, Miller KD, \& Jemal A (2019). Cancer statistics, CA: Cancer J. Clin, 69, 734.https://doi.org/10.3322/caac.2155.

2. Siegel RL, Miller KD, Fuchs HE, \& Jemal A (2021) Cancer statistics, CA: Cancer J. Clin, 71, 7-33. https://doi.org/10.3322/caac. 21654.

3. WHO (2019) Cancer statistics, https://www.who.int/health-topics/cancer\#tab=tab_1.

4. Schlessinger J, \& Ullrich A. (1992) Growth factor signaling by receptor tyrosine kinases. Neuron, 9, 383-391. https://doi.org/10.1016/0896-6273(92)90177-f.

5. Hunter, T. Signaling (2000) and beyond. Cell 100, 113-127. https://doi.org/10.1016/s0092-8674(00)81688-8.

6. Park JH, Liu Y, Lemmon MA and Radhakrishnan R. (2012) Erlotinib binds both inactive and active conformations of the EGFR tyrosine kinase domain. Biochem J, 448(Pt 3), p.417 https://dx.doi.org/10.1042\%2FBJ20121513.

7. Landi L, \& Cappuzzo F (2013) HER2 and lung cancer. Expert Rev. Anticancer Ther, 1219-1228. https://doi.org/10.1586/14737140.2013.846830

8. Sharma SV, Bell DW, Settleman, J et al (2007). Epidermal growth factor receptor mutations in lung cancer. Nat. Rev. Cancer, 7, 169-181. https://doi.org/10.1038/nrc2088.

9. Woo HS., Ahn HK et al (2014) Epidermal growth factor receptor (EGFR) exon 20 mutations in non-small-cell lung cancer and resistance to EGFR-tyrosine kinase inhibitors. Investig. New Drugs, 32, 13111315.https://doi.org/10.1007/s10637-014-0146-x. 
10. Huang L, \& Fu L (2015) Mechanisms of resistance to EGFR tyrosine kinase inhibitors. Acta Pharm. Sin. B. 2015, 5, 390-401. https://doi.org/10.1016/j.apsb.2015.07.001

11. Heald R, Bowman, KK, Bryan MC et al (2015). Noncovalent mutant selective epidermal growth factor receptor inhibitors: a lead optimization case study. J. Med. Chem, 58, 8877-

8895.https://doi.org/10.1021/acs.jmedchem.5b01412.

12. Chan, et al (2016) Heffron, T. P. Discovery of a noncovalent, mutant-selective epidermal growth factor receptor inhibitor. J. Med. Chem. 59, 9080-9093.https://doi.org/10.1021/acs.jmedchem.6b00995.

13. Yun $\mathrm{CH}$, Mengwasser KE, Toms AV et al (2008). The T790M mutation in EGFR kinase causes drug resistance by increasing the affinity for ATP. PNAS, 105, 2070-2075. https://doi.org/10.1073/pnas.0709662105

14. Wang Y, Suh YA et al (2011). Restoring expression of wild-type p53 suppresses tumor growth but does not cause tumor regression in mice with a p53 missense mutation. J.Clin.Investig 121, 893904 https://doi.org/10.1172/jci44504.

15. Holohan C, Van Schaeybroeck S, Longley DB et al (2013). Cancer drug resistance: an evolving paradigm. Nat. Rev. Cancer 13, 714-726.https://doi.org/10.1038/nrc3599.

16. Ammar YA (2018). Design, synthesis, antiproliferative activity, molecular docking and cell cycle analysis of some novel (morpholinosulfonyl) isatins with potential EGFR inhibitory activity. European J. Med. Chem.,156, 918-932. https://doi.org/10.1016/j.ejmech.2018.06.061.

17. Hanwell MD, Curtis DE, Lonie DC et al (2012) Avogadro: an advanced semantic chemical editor, visualization, and analysis platform. J cheminformatics, 4, 1-17.https://doi.org/10.1186/1758-2946-4-17.

18. El-Azab et al (2010). Design, synthesis and biological evaluation of novel quinazoline derivatives as potential antitumor agents: molecular docking study. Eur. J. Med. Chem. 45, 4188-4198.

https://doi.org/10.1016/j.ejmech.2010.06.013.

19. El-Deeb et al (2010) Synthesis and antitumor evaluation of novel cyclic arylsulfonylureas: ADME-T and pharmacophore prediction. European J. Med. Chem 45, 2516-2530.

https://doi.org/10.1016/j.ejmech.2010.02.038

20. Stamos et al (2002). Structure of the epidermal growth factor receptor kinase domain alone and in complex with a 4-anilinoquinazoline inhibitor. J Biol Chem, 277 46265-46272.https://doi.org/10.1074/jbc.m207135200.

21. Thompson MA, (2004) Molecular docking using ArgusLab, an efficient shape-based search algorithim and AScore scoring function, in proceedings of the ACS Meeting, Philadelphia, Pa, USA, March- April 2004, 172, CINF 42.

22. Morris GM, Huey R, Lindstrom W et al (2009) AutoDock4 and AutoDockTools4: Automated docking with selective receptor flexibility.J. Comput. Chem 30, 2785-2791.https://doi.org/10.1002/jcc.21256.

23. Jo S, Kim T, lyer VG et al (2008) CHARMM-GUI: a web-based graphical user interface for CHARMM.J. Comput. Chem, 29, 1859-1865 https://doi.org/10.1002/jcc.20945.

24. Pereira GRC, Da Silva ANR et al (2019). In silico analysis and molecular dynamics simulation of human superoxide dismutase 3 (SOD3) genetic variants. J. Cell.Biochem120, 2019, 3583-

3598. https://doi.org/10.1002/jcb.27636.

25. Vanommeslaeghe K, Hatcher E, Acharya C et al (2010) CHARMM general force field: A force field for drug-like molecules compatible with the CHARMM all-atom additive biological force fields. J. Comput. Chem, 2010, 31, 671-690.https://doi.org/10.1002/jcc.21367. 
26. Vanommeslaeghe K, \& MacKerell Jr, AD. (2012). Automation of the CHARMM General Force Field (CGenFF) I: bond perception and atom typing. J Chem Inf Model, 52, 3144-3154. https://doi.org/10.1021/ci300363c.

27. Vanommeslaeghe, K., Raman, E. P., \& MacKerell Jr, A. D. (2012). Automation of the CHARMM General Force Field (CGenFF) II: assignment of bonded parameters and partial atomic charges. J Chem Inf Model, 52 31553168. https://doi.org/10.1021/ci3003649

28. Huang J., \& MacKerell Jr, AD. (2013). CHARMM36 all-atom additive protein force field: Validation based on comparison to NMR data. J. Comput. Chem 34, 2135-2145. http://doi.org/10.1002/jcc.23354.

29. MarkP, \& Nilsson L (2001). Structure and dynamics of the TIP3P, SPC, and SPC/E water models at $298 \mathrm{~K}$. J. Phys. Chem. A, 2001 105, 9954-9960.https://doi.org/10.1021/jp003020w.

30. Borkotoky S, \& Murali A. (2018). A computational assessment of pH-dependent differential interaction of T7 lysozyme with T7 RNA polymerase. BMC Struct Biol, 17, 1-11. https://doi.org/10.1186/s12900-017-0077-9.

31. Hess B, Bekker H, Berendsen, HJ, et al (1997). LINCS: a linear constraint solver for molecular simulations. J. Comput. Chem,1997, 18, 1463-1472. https://doi.org/10.1021/ct5007983.

32. Brooks, BR., Brooks III CL, Mackerell Jr, AD et al (2009) CHARMM: the biomolecular simulation program. J. Comput. Chem, 30, 1545-1614. http://doi.org/10.1002/jcc.21287.

33. R Kumari R Kumar, (2014) Open-Source Drug Discovery Consortium and Lynn, A. g_mmpbsa. A GROMACS tool for high-throughput MM-PBSA calculations. J. chem. Inf. model, 54, 1951-1962. https://doi.org/10.1021/ci500020m.

34. Pires DE, Blundell TL, \& Ascher DB (2015). pkCSM: predicting small-molecule pharmacokinetic and toxicity properties using graph-based signatures. J. Med. Chem, 58, 4066-4072.

https://doi.org/10.1021/acs.jmedchem.5b00104.

35. Molsoft LLC (2007). 3366, North Torrey Pines Court, Suite 300, La Jolla, CA 92037, USA

36. Daina A, Michielin O, \& Zoete, V (2017) SwissADME: a free web tool to evaluate pharmacokinetics, druglikeness and medicinal chemistry friendliness of small molecules. Sci. Rep, 7, 113.https://doi.org/10.1038/srep42717.

37. O'Boyle NM, Banck M, James CA (2011) Open Babel: An open chemical toolbox. J cheminformatics, 3, 114.https://doi.org/10.1186/1758-2946-3-33.

38. Kitazaki T, Oka M, Nakamura Y, Tsurutani J, et al (2005). Gefitinib, an EGFR tyrosine kinase inhibitor, directly inhibits the function of P-glycoprotein in multidrug resistant cancer cells. Lung Cancer, 49, 337-343. https://doi.org/10.1016/j.lungcan.2005.03.035.

39. El-Sayed S, Metwally K, El-Shanawani AA et al (2017). Synthesis and anticancer activity of novel quinazolinone-based rhodanines. Chem. Cent. J., 11, 1-10. http://doi.org/10.1186/s13065-017-0333-x.

40. Xu W, Jin Y, Liu H, Jiang Y, \& Fu AH. (2011). Copper-Catalyzed Domino Synthesis of Quinazolinones via Ullmann-Type Coupling and Aerobic Oxidative C- H Amidation. Org Lett, 13, 1274-1277. https://doi.org/10.1021/jo101685d.

41. Pericherla., Khedar P, Khungar B, \& Kumar A. (2013). One-pot sequential C-N coupling and cross dehydrogenative couplings: synthesis of novel azole fused imidazo [1, 2-a] pyridines.Chem Comm, 49, 29242926.https://doi.org/10.1007/s11030-015-9646-7.

42. Sadhasivam A, Nagarajan H, \& Umashankar V (2020). Structure-based drug target prioritisation and rational drug design for targeting Chlamydia trachomatis eye infections. J. Biomol. Struct. Dyn, 38, 3131-3143. https://doi.org/10.1080/07391102.2019.1652691.

Page $15 / 24$ 
43. Sharma J, Bhardwaj VK, Das P et al (2021). Identification of naturally originated molecules as Y-aminobutyric acid receptor antagonist. J. Biomol. Struct. Dyn, 39, 911-

922. https://doi.org/10.1080/07391102.2020.1720818

44. Goyal S, Jamal S, Shanker A, Grover A (2015). Structural investigations of T854A mutation in EGFR and identification of novel inhibitors using structure activity relationships. BMC genomics,16(5), pp.1-9.

https://doi.org/10.1186/1471-2164-16-S5-S8.

45. Li DD, Wu TT, Yu P, Wang ZZ et al (2020). Molecular dynamics analysis of binding sites of epidermal growth factor receptor kinase inhibitors. ACS omega,5(26), pp.16307-16314

https://doi.org/10.1021/acsomega.0c02183.

46. Kim MK, Yee J, Cho YS, Jang HW, Han JM, Gwak HS (2018) Risk factors for erlotinib-induced hepatotoxicity: a retrospective follow-up study. BMC Cancer. 18(1) pp. 1-7. https://doi.org/10.1186/s12885-018-4891-7.

\section{Figures}

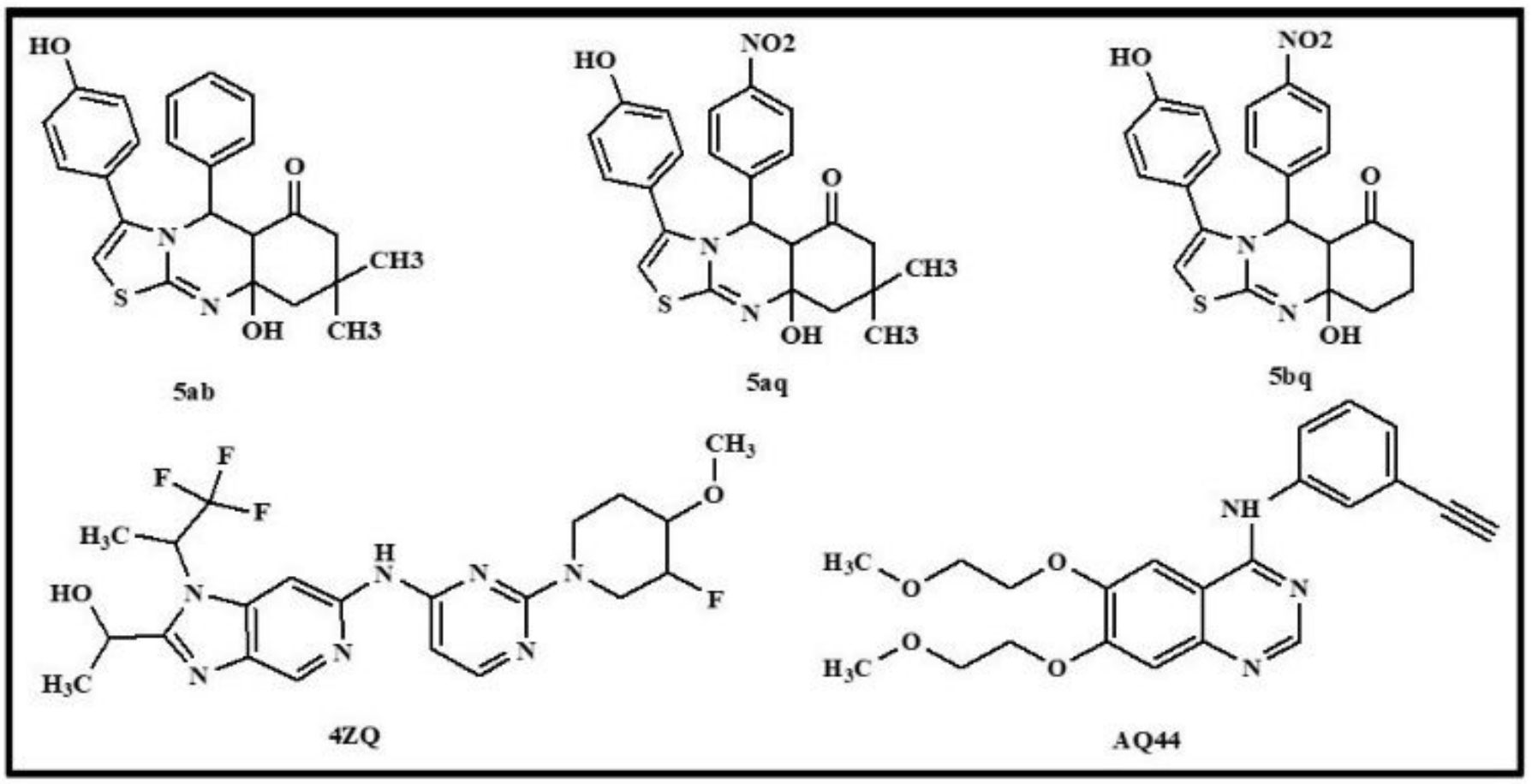

Figure 1

2D Structures of thiazolo-[2,3-b] quinazolinone derivatives (5ab, 5aq and $5 \mathrm{bq}$ ) and reference ligands of wtEGFRTKD (AQ44) and TMLR EGFR-TKD mutant “TMLR” (4ZQ). 


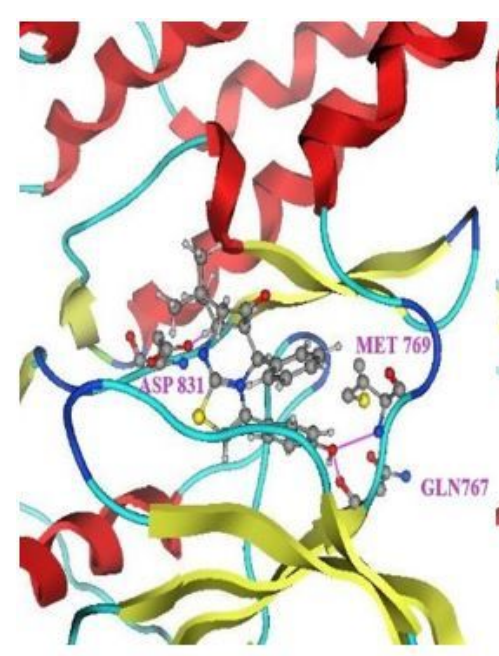

$5 \mathbf{a b}$

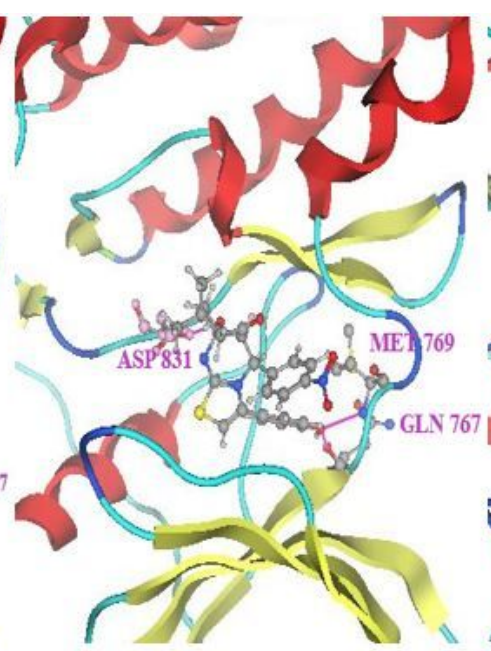

5 aq

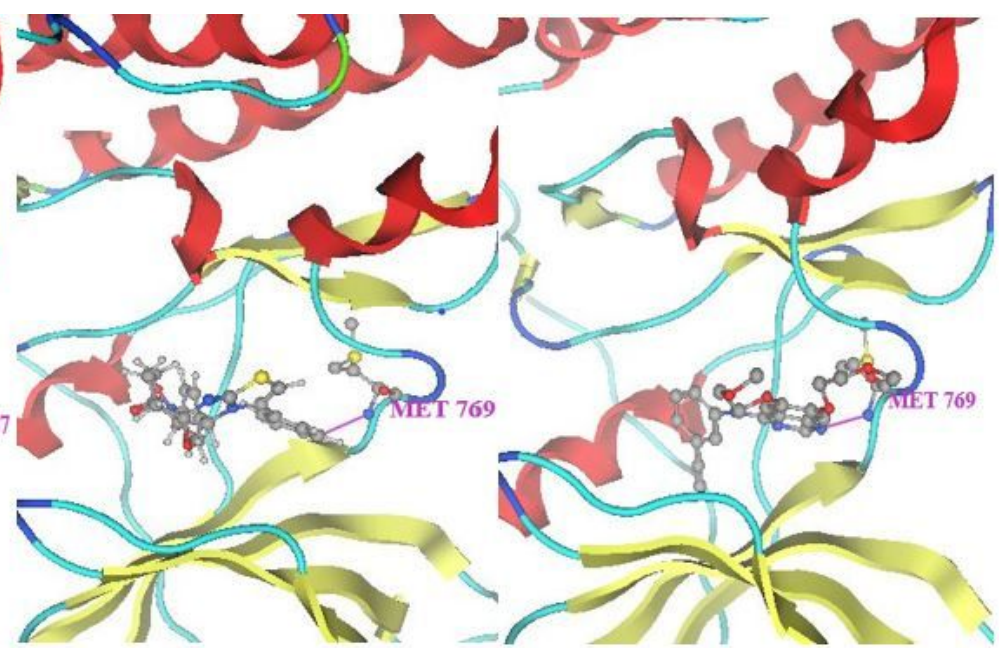

$5 \mathbf{b q}$
Erlotinib

Figure 2

Molecular interactions by thiazolo-[2,3-b] quinazolinone derivatives (5ab, 5aq and 5bq) and reference ligand erlotinib (AQ44) with catalytic site of wtEGFR-TKD.

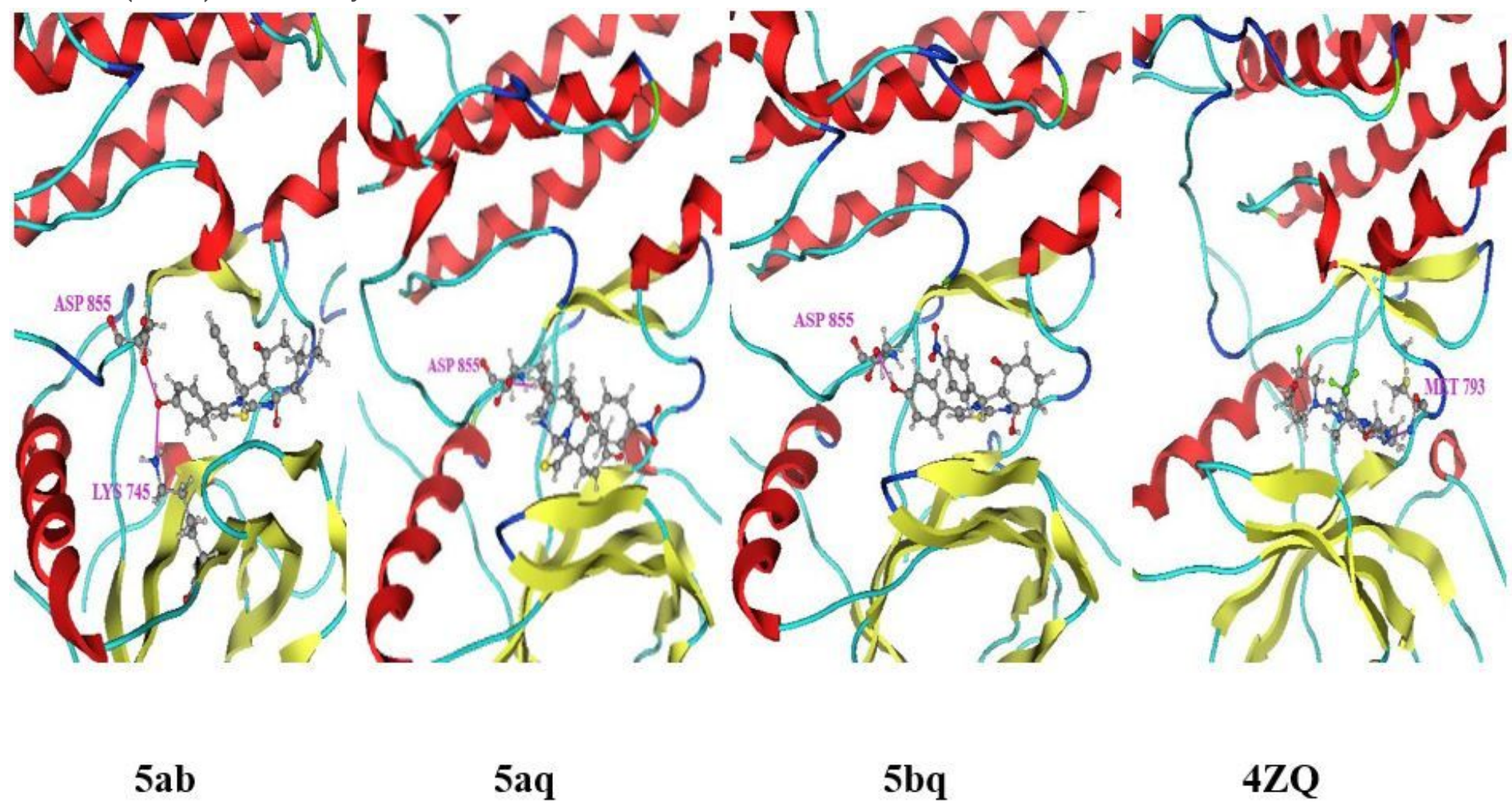

Figure 3

Molecular interactions exploited by thiazolo [2,3-b] quinazolinones (5ab, 5aq and 5bq) and reference ligand 4ZQ with catalytic site of EGFR-TKD mutant "TMLR". 

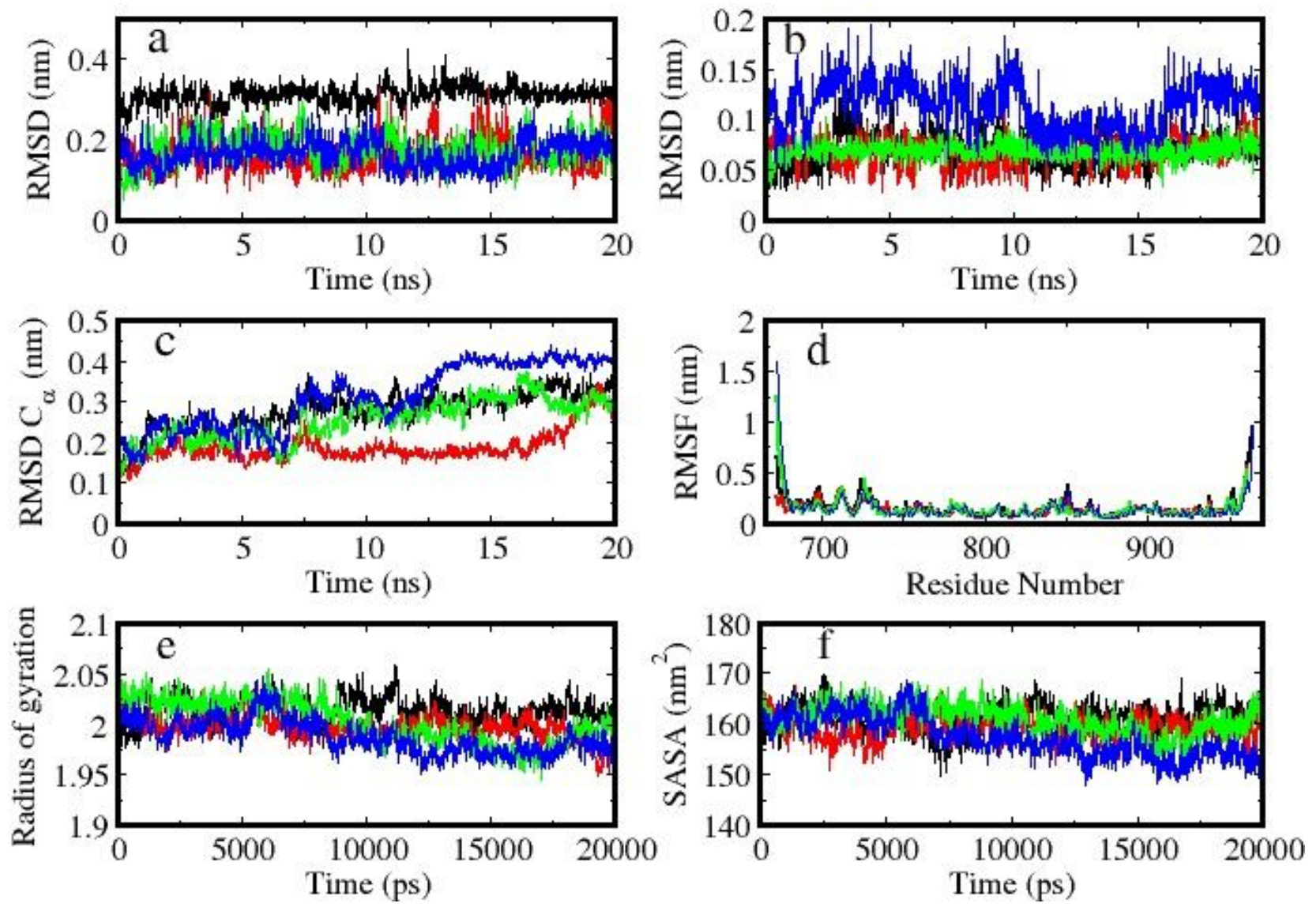

Figure 4

Trajectories of thiazolo-[2,3-b] quinazolinone derivatives-1M17 complexes and AQ44-1M17 complex are represented by colors $5 a b=b l a c k, 5 a q=r e d, 5 b q=g r e e n$ and AQ44= blue, (a) RMSD of ligand movement (b) ligand conformations (c) RMSD of $\mathrm{Ca}$ (d) RMSF of Protein (e) Radius of gyration and (d) Solvent accessible surface area in aqueous medium. 

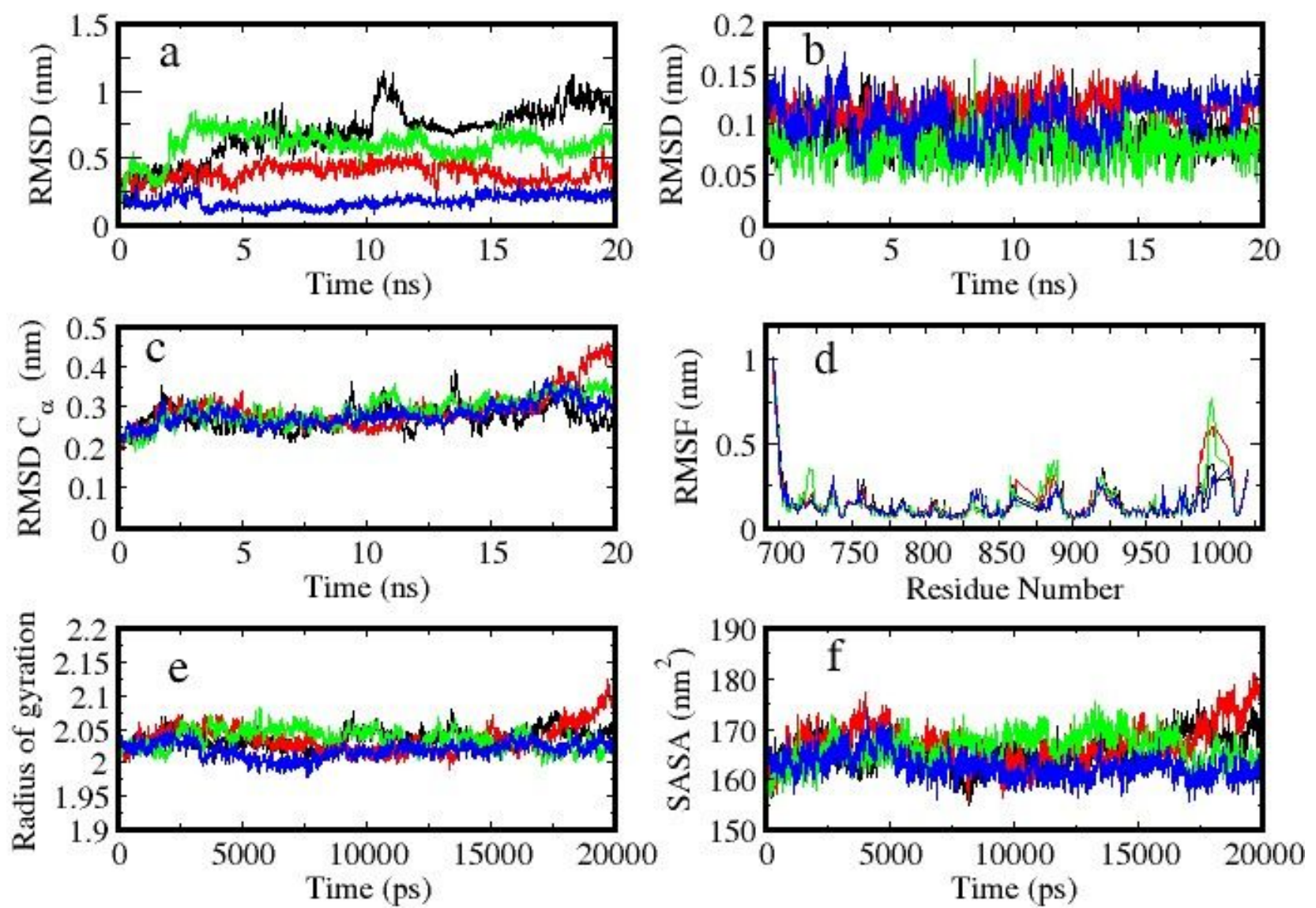

Figure 5

Trajectories of thiazolo-[2,3-b] quinazolinone derivative-5CAS complexes, 4ZQ-5CAS complex are represented by colors 5ab=black, 5aq=red, 5bq=green and AQ44= blue, (a) RMSD of ligand movement (b) ligand conformations (c) RMSD of Ca (d) RMSF of Protein (e) Radius of gyration and (d) Solvent accessible surface area for 20ns of simulation in aqueous medium. 

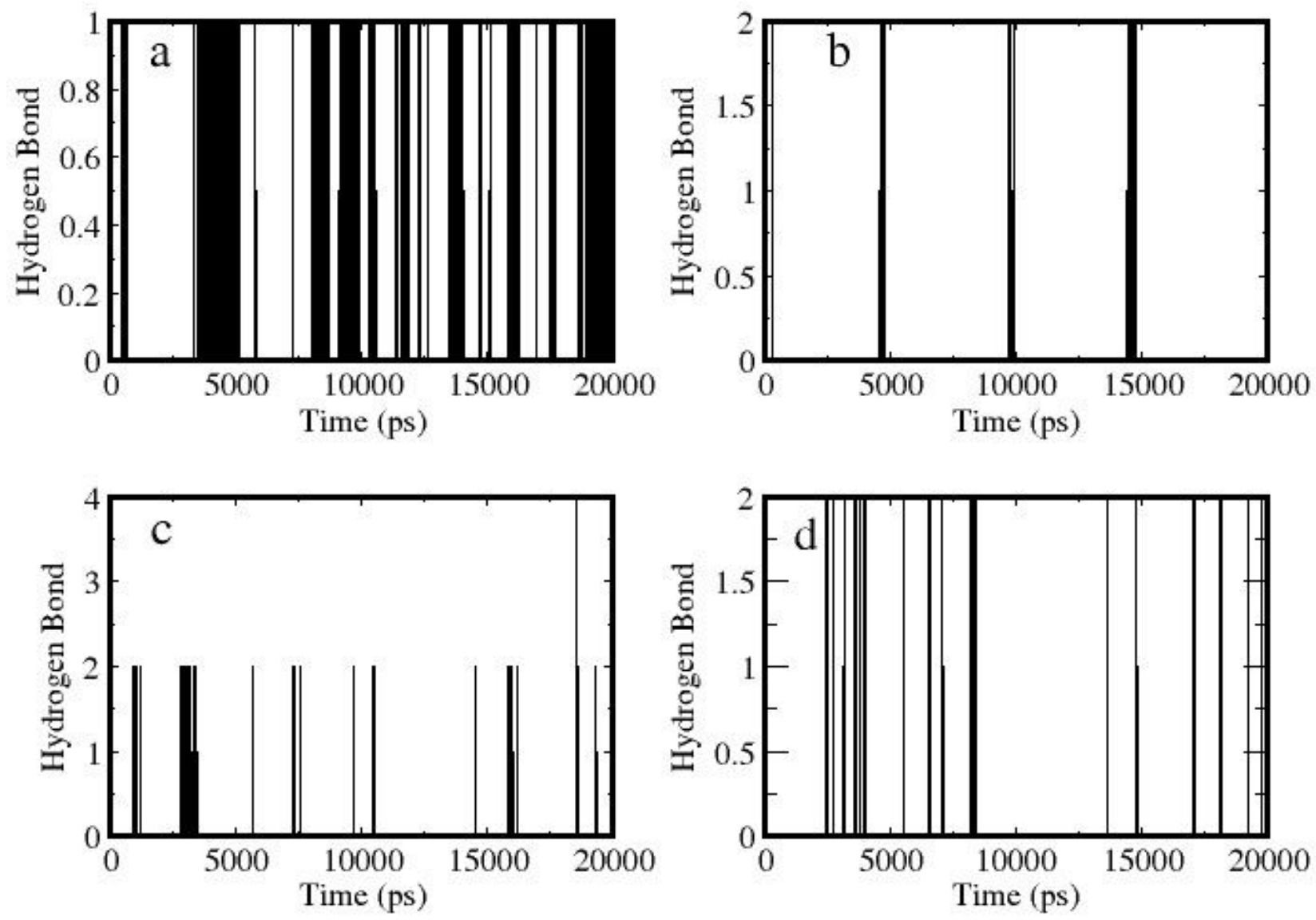

Figure 6

Hydrogen bond formed by thiazolo-[2,3-b] quinazolinone derivatives and AQ44 with wtEGFR-TKD complexes are presented as (a) 5ab, (b) 5ab, (c) 5ab and (d)AQ44. 

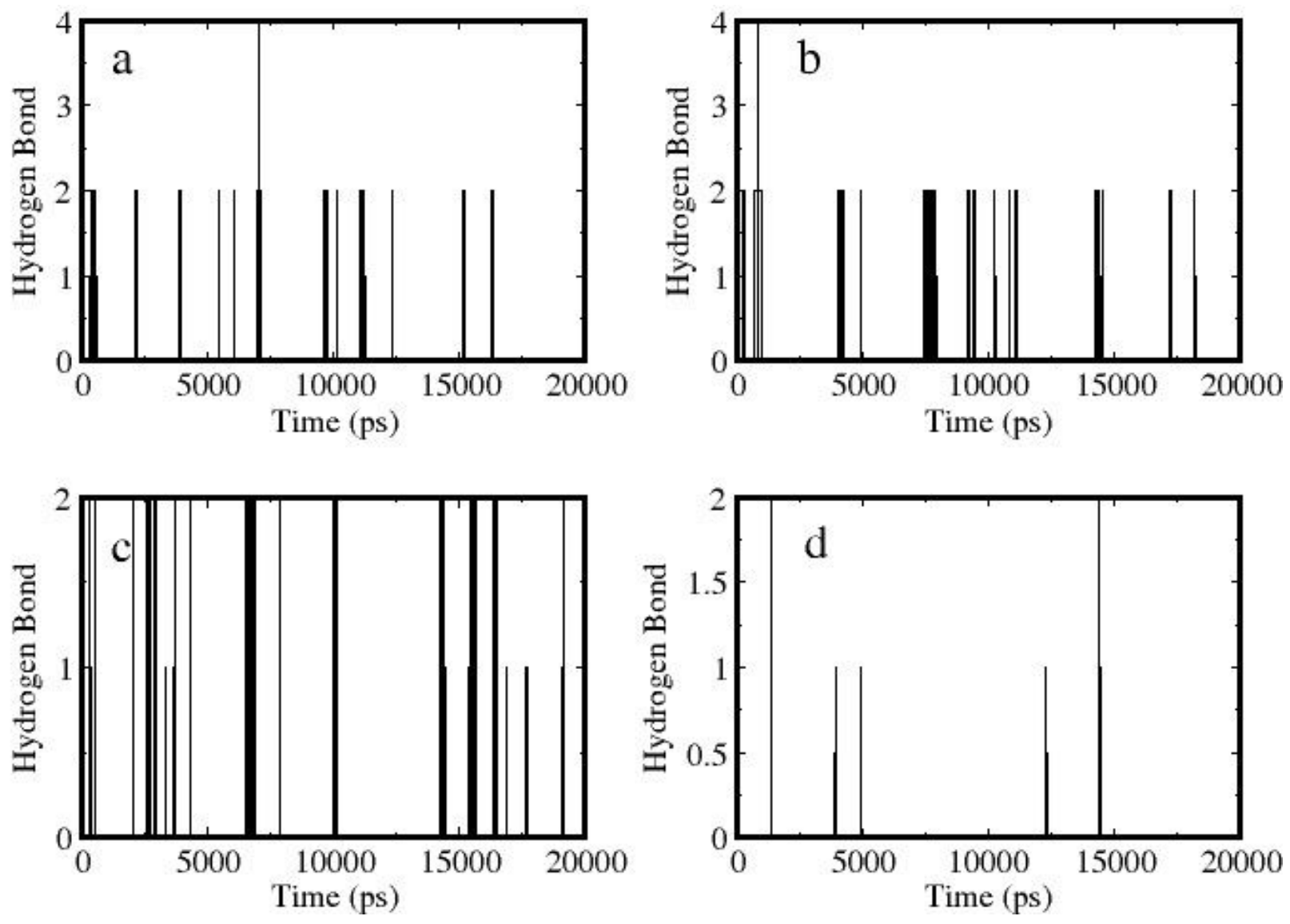

Figure 7

Hydrogen bond formed by thiazolo-[2,3-b] quinazolinone derivatives and 4ZQ with EGFR-TKD mutant "TMLR" are presented as (a) 5ab, (b) 5ab, (c) 5ab and (d) 4ZQ. 

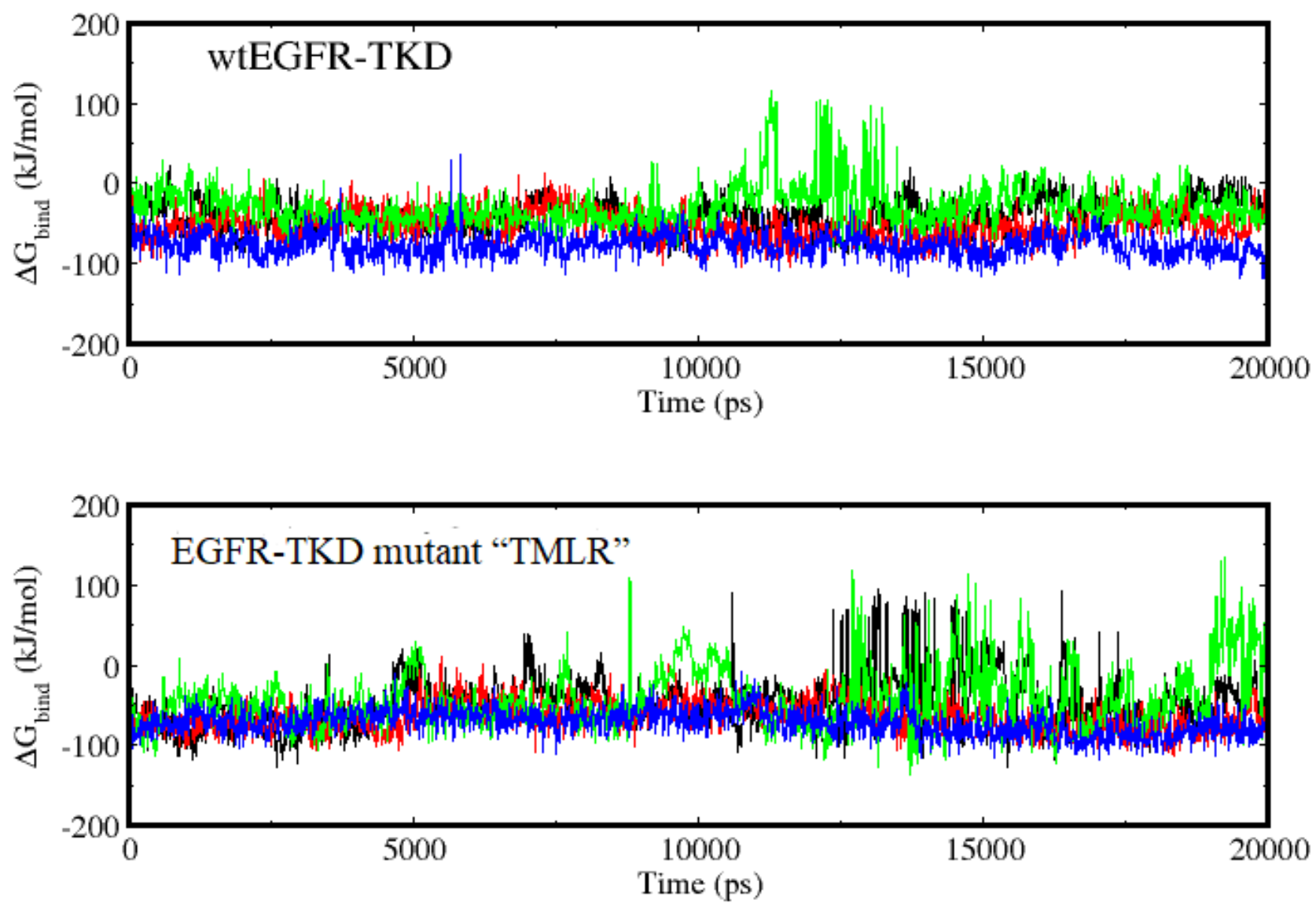

Figure 8

The binding energy of thiazolo-[2,3-b] quinazolinone derivatives with wtEGFR-TKD complexes is mentioned in upper along with reference ligand complex and binding energy of thiazolo-[2,3-b] quinazolinone derivatives with EGFRTKD mutant "TMLR" complexes were given in below panel, the color representations were given as 5ab black, $5 a q$ red, $5 \mathrm{bq}$ green and reference ligands blue. 


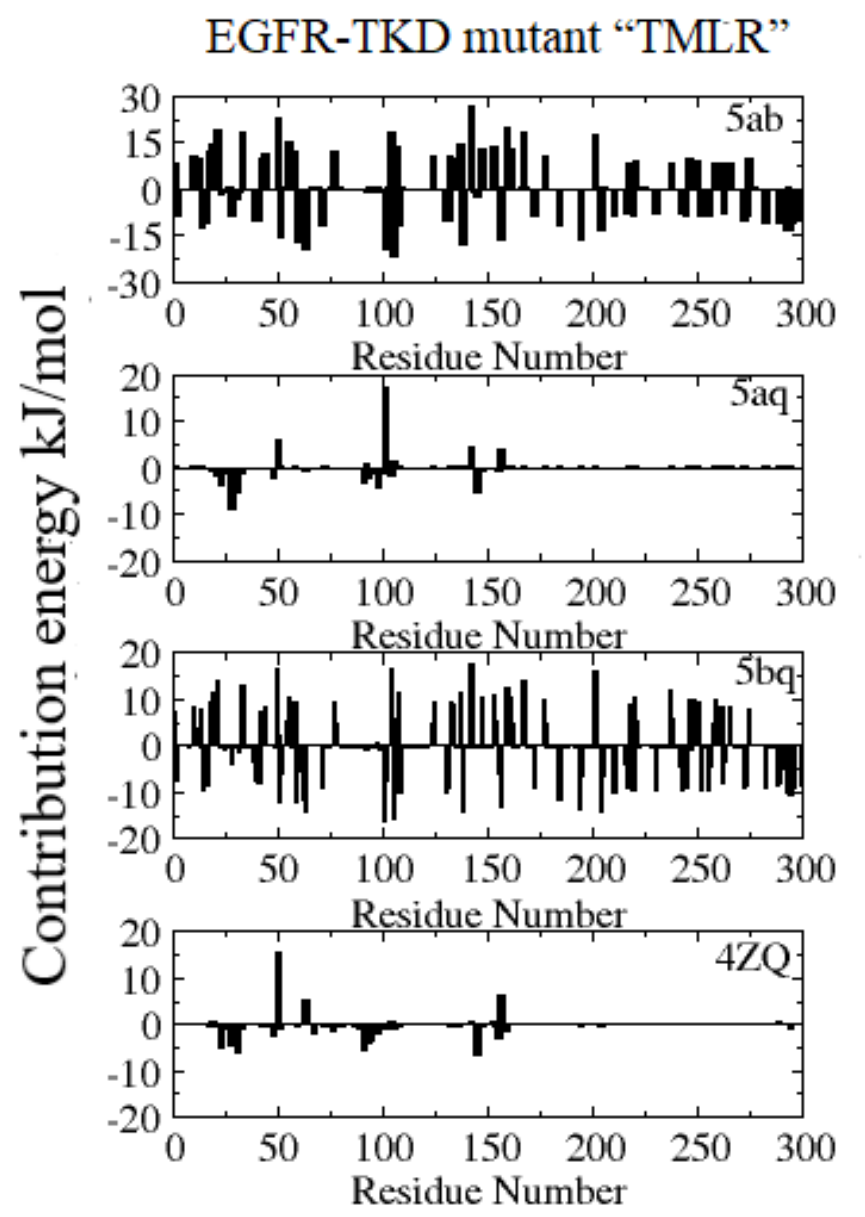

wtEGFR-TKD
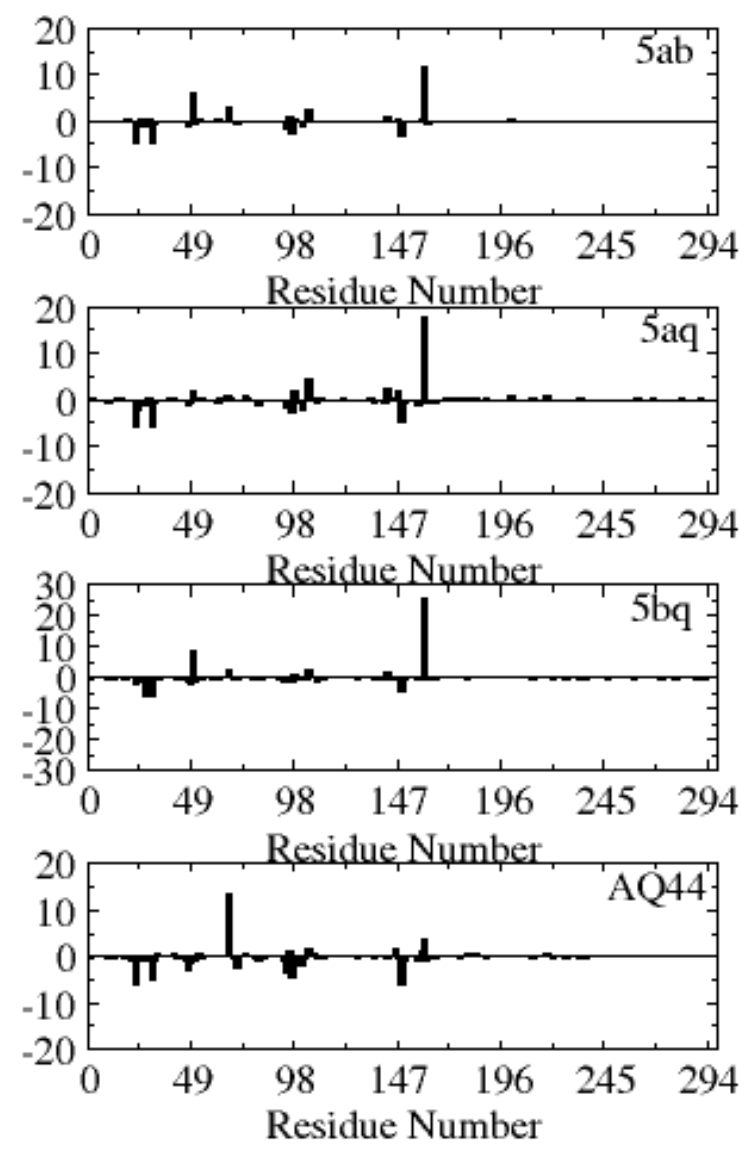

Figure 9

The contribution by amino acids in free energy decomposition were plotted, left row represents wtEGFR-TKD and right row EGFR-TKD mutant "TMLR" 

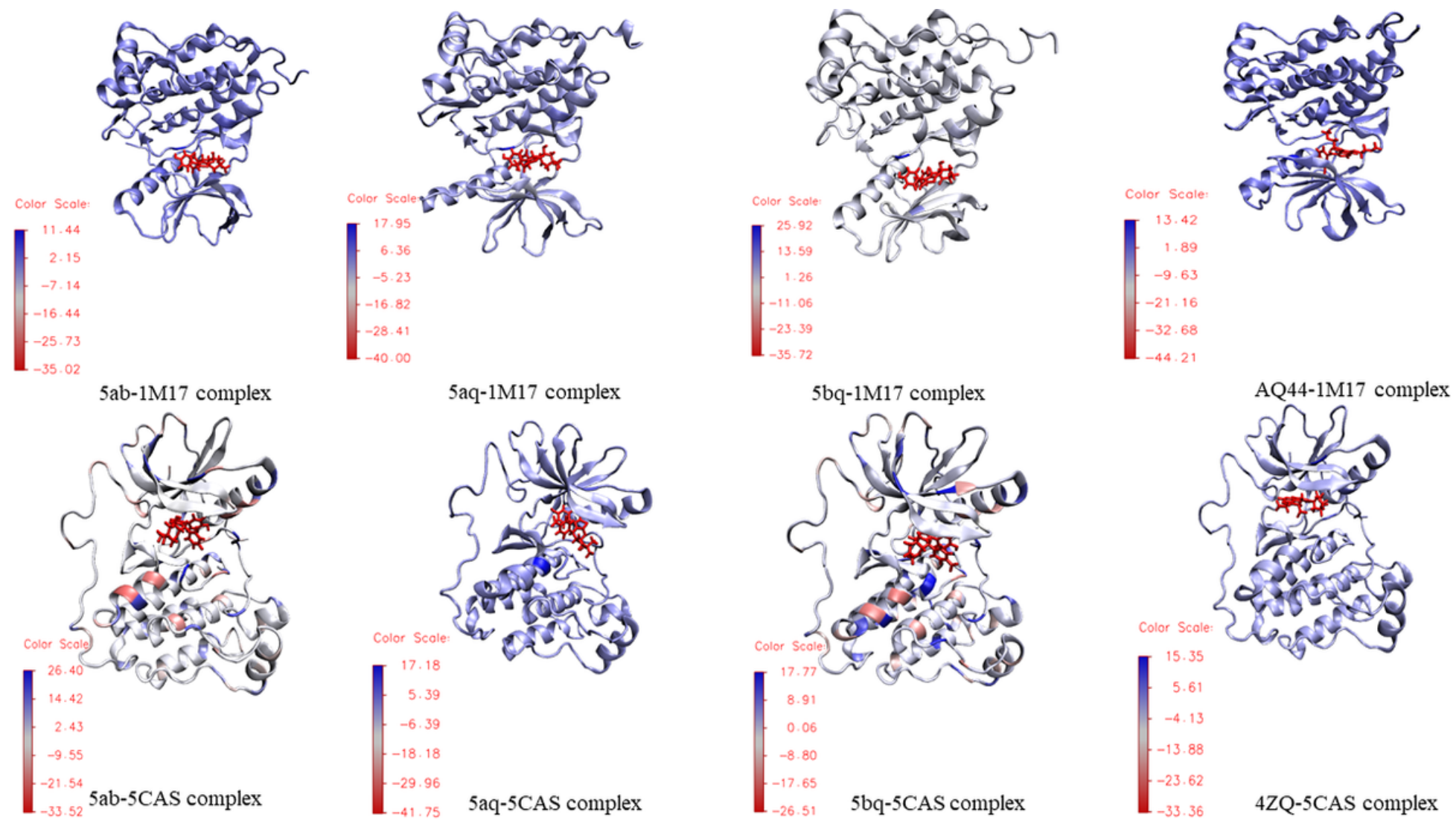

Figure 10

The upper panel complexes are represented as wtEGFR-TKD- thiazolo-[2,3-b] quinazolinone derivatives (5ab,5aq,5bq), AQ44 and below panel complexes are represented as EGFR-TKD mutant "TMLR". The ligand is annotated in red colored and green turns and loops of both EGFR-TKD contributes in free energy decomposition. Color bar scale represents the energy decomposition by both proteins and ligands.

\section{Supplementary Files}

This is a list of supplementary files associated with this preprint. Click to download.

- GraphicalAbstract.jpg

- JMMSuppl.docx 